\title{
V. HERZOGIN VON GUISE
}

\section{1. »Mon devoir en l'absence de Monsieur mon mary« \\ Die Verwalterin}

\section{Protagonisten und Schauplätze}

Im April 1550 war Claude de Lorraine gestorben. Anna, die nun den Titel der Herzogin von Guise trug, verbrachte fortan einen großen Teil ihrer Zeit in Joinville in Gesellschaft der Schwiegermutter. Antoinette de Bourbon, von einem italienischen Beobachter als zwar alte, aber fresca donna bezeichnet, hatte sich nach dem Tod ihres Gemahls in das dortige Schloß zurückgezogen, um die Familienangelegenheiten zu überwachen und ihre Enkelkinder zu erziehen. Die in einem Inventar vermerkten grands ciels de lit en tapisserie de haute lice aux chiffres de François de Guise et d'Anne d'Este zeigen, daß auch das neue Herzogspaar seinen festen Platz in Joinville hatte. Von hier schrieb Anna dem Vater: Io sono qui a Ginvila con Madama di Guisa, la qual prometto a V.E. mette ogni pena che gli è possibile a tratarmi meglio assai ch'io non merto. Das Verhältnis der beiden Damen scheint tatsächlich sehr gut gewesen zu sein. Konnte Anna nicht in Joinville sein, wurde sie von Antoinette über Neuigkeiten unterrichtet, und in den stets mit Ma fille, ma mie überschriebenen Briefen finden sich nicht selten Stellen, die dem Wunsch der Witwe Ausdruck verliehen, möglichst häufig alle ihre Kinder und Enkelkinder um sich zu haben'.

Unkompliziert gestaltete sich auch Annas Beziehung zu den Geschwistern ihres Gemahls. Renée, Äbtissin des Klosters Saint-Pierre in Reims, bezeichnete ihre Schwägerin als petite mignonne; François, grand prieur de France, schickte ihr einen letzten Gruß vor seiner Abfahrt nach Malta und bat sie, me conserver tousjours en votre bonne grace, und das Verhältnis zu Louis, Kardinal von Guise, spiegelt sich schließlich in zahlreichen Briefen wider, die er

${ }^{1}$ Giovanni Michiel, Relazione für die Serenissima, 1561, in: TOMMASEO (Hg.), Relations, Bd. 1, S. 436; Inventar des Schlosses von Joinville, 15. März 1583, in: Émile HUMBLOT, Roger LUZU, Les Seigneurs de Joinville, Saint-Dizier 1964, S. 245; Anna d'Este an Ercole d'Este, 3. Sept. 1549, Joinville, in: ASM, Canc. duc., Cart. prin. est. 1568 A/14; Antoinette de Bourbon an Anna d'Este, 11. März (nicht Mai!) (1549), in: PIMODAN, Antoinette de Bourbon, S. 263. Zu dem als »Grand Jardin« oder »Château d'en bas« bezeichneten Schloß: Émile HuMBLOT, Le Château du Grand Jardin 1546. Maison de plaisance de Claude de Lorraine et d'Antoinette de Bourbon à Joinville, in: Mémoires de la Société des lettres, des sciences, des arts, de l'agriculture et de l'industrie de Saint-Dizier 10/1 (1906) S. 1-71. 
ihr im Laufe seines Lebens schrieb ${ }^{2}$. Die kleine Maria Stuart, Tochter der Schwester des Herzogs von Guise, verbrachte viel Zeit in den Hăusern und Schlössern der Familie, wo sie von François und seiner Gemahlin wie ihr eigenes Kind behandelt wurde ${ }^{3}$. Eine wirkliche Freundschaft verband Anna mit Charles de Lorraine, Kardinal von Lothringen, von Paolo Giovio als maraviglioso giovine beschrieben und fur seine reichen Geistesgaben gelobt; doch wurden ihm auch Geiz, Gier, Unehrlichkeit und Rachsucht vorgeworfen, außerdem eine Persönlichkeit prontissima all' offendere ${ }^{4}$. Die Zuneigung der Prinzessin zu ihrem Schwager läßt sich an der Schenkung ablesen, die Anna kurz vor der Geburt ihres ersten Kindes zu seinen Gunsten tätigte, ähnlich der von vielen Frauen vor einer Niederkunft veranlaßten Regelungen. Wie in einem Testament bedachte die gerade Achtzehnjăhrige den Kardinal mit allen Schmuckstucken und Edelsteinen, die sich zum Zeitpunkt ihres Todes in ihrem Besitz befinden wurden, sy de son mariage elle n'a aucuns enfans lors dicelluy ${ }^{5}$. Charles schrieb seiner Schwägerin regelmäßig. Aus seinen Briefen spricht die Sorge um Annas Wohlergehen und der Wunsch, die Prinzessin möglichst oft zu sehen: il n'i a remede, il fault que je vous voye. Manchmal, wenn er sich in Gesellschaft des Herzogs von Guise befand, fugte letzterer einen kurzen Gruß an die Gattin hinzu' ${ }^{6}$. Wenn sie nicht des Krieges oder anderer Gründe wegen getrennt waren, bildeten Anna, François und Charles ein wahres Dreigespann, wie auch Katharina von Medici, in politisch schwierigen Zeiten nicht immer ohne Bedauern, feststellen mußte: vostre bon mari et bon frère et vous n'este que heun come la Trinité 7 .

Neben Joinville gehörten Paris und Nanteuil zu den bevorzugten Aufenthaltsorten der Prinzessin. Im Juni 1553 hatten Anna und François mit dem

${ }^{2}$ Renée de Lorraine an François de Lorraine, o.D., in: BnF, Clair. 345, S. 281; François de Lorraine, grand prieur de France, an Anna d'Este, 18. Dez. 1553, Marseille, in: BnF, Clair. 347, fol. 148r. Die Briefe von Louis de Lorraine, nicht zu verwechseln mit denen seines gleichnamigen Neffen, befinden sich v.8. in: BnF, Fr. 3231.

${ }^{3}$ Maria Stuart an Marie de Lorraine, 28. Dez. 1555, 22. März 1557, Mai 1557, in: Marie STUART, reine d'Écosse, Lettres, instructions et mémoires, hg. v. Alexandre LABANOFF, Bd. 1, London 1844, S. 32, 37, 44f.

${ }^{4}$ Paolo Giovio an Ippolito d'Este, o.D. (Jan. 1548 aus Rom), in: Paolo Giovio, Lettere, Bd. 2, hg. v. Guiseppe Guido FERRERO, Rom 1958, S. 119; Giovanni Michiel, Relazione für die Serenissima, 1561, in: TOMMASEO (Hg.), Relations, Bd. 1, S. 438.

'Donnation non signée faict par dame Anne d'Est à Monseigneur le cardinal de Guise, in: Inventaire Joinville, fol. 17r. Zu den weiblichen Angehörigen des venezianischen Adels, die kurz vor einer Niederkunft ihr Testament verfaßten: Volker HUNECKE, Der venezianische Adel am Ende de Republik, 1646-1797. Demographie, Familie, Haushalt, Tübingen 1995, S. 96f.; Anna BELlAVITIS, Dot et richesse des femmes à Venise au XVI siècle, in: Gabrielle HoUBRE (Hg.), Femmes, dots et patrimoines, Toulouse 1998, S. 91-100, hier S. 97.

$6 \mathrm{Vgl}$. die zahlreichen Briefe in: Lorraine, Lettres. Das Zitat aus dem Brief vom 15. Jan. 1564, in: Ibid. S. 509.

${ }^{7}$ Katharina von Medici an Anna d'Este, Dez. 1561, in: MÉDICIS, Lettres, Bd. 1, S. 259. 
Erwerb des späteren Hôtel de Guise begonnen. Das Herzogspaar kaufte zunăchst eine grande maison, contenant plusieurs corps d'hostel, estables, cours et jardins, gelegen in der Rue du Chaume, heute Rue des Archives, in dem als Marais bezeichneten Stadtviertel von Paris. Hinzu kamen in den folgenden Jahren weitere, in unmittelbarer Nachbarschaft befindliche Hăuser und Grundstücke, so daß die Familie Anfang der 1560er Jahre ein großes Areal in der Năhe des Temple ihr Eigen nennen konnte ${ }^{8}$. Die Gegend gehörte in der zweiten Hälfte des 16. Jahrhunderts zu den begehrtesten der Stadt, adlige Familien wie die Montmorency und viele königliche Beamte wohnten hier, und die Steuern waren höher als in anderen Stadtteilen. Gegen Ende des 14. Jahrhunderts hatte das Gelände an der Rue du Chaume, wo sich später das Hôtel de Guise erheben sollte, dem Konnetabel Olivier Clisson gehört, der darauf ein Gebăude errichtete, dessen mit zwei Türmen versehenes Tor noch heute gegenüber der Rue de Braque zu bewundern ist. Die Guise ließen das Wohnhaus vergrößern, die grant salle haulte renovieren, und nach Plänen des Italieners Francesco Primaticcio eine Kapelle bauen, von der sich nur ein Teil der Fassade erhalten hat. Der Freskenschmuck, ausgeführt von Niccolò dell' Abbate, und die in der Akte eines Notars erwähnten deux imaiges, l'une saint Françoys et l'autre sainte Anne, sind verloren. Zu Beginn des 18. Jahrhunderts wurde der Komplex zum Hôtel de Soubise umgebaut, heute ist das Haus Sitz des französischen Nationalarchivs'.

Auf einem Plan der Stadt Paris vom Beginn des 17. Jahrhunderts sind die Ausmaße des Hôtel de Guise zu erkennen, das neben dem eigentlichen Wohngebäude vielerlei Ställe und Werkstătten, Höfe und Gärten einschloß (Abb. 3). Die Ausgaben für die Instandhaltung eines Anwesens dieser Größe waren enorm. Achtzig Livres erhielt allein der Gärtner, tant pour les fruictz qui estoient au jardin [...] que pour la valleur des arbres qu'il a planté au par-

${ }^{8}$ Kaufvertrag zwischen Anna d'Este und François de Lorraine auf der einen und Philibert Babou de La Bourdaisière auf der anderen Seite, 14. Juni 1553, in: BnF, Fr. 22389, fol. 35f. Das Zitat nach: LANGLOIS, Les hôtels, S. 12, Anm. 1. Vgl. das Inventar des Archivs des Hôtel de Guise, 1688, in: L. BRIĖLE (Hg.), Collection de documents pour servir à l'histoire des hôpitaux de Paris, Bd. 4: Fin des comptes et dons et legs faits avant 1789 aux hôpitaux et hospices, Paris 1887, S. 199f.; Jules QUICHERAT, La porte de l'hôtel Clisson, in: Revue archéologique 4 (1848) S. 760-769, hier S. 763f. Zum Stadtviertel rund um den Temple: Barbara B. DIEFENDORF, Paris City Councillors in the Sixteenth Century. The Politics of Patrimony, Princeton 1983, S. 61.

9 Aktenregister der Pariser Notare, 5. Nov. 1554, 14. Mărz 1555, in: Catherine GRODECKI, Documents du Minutier central des notaires de Paris. Histoire de l'art au XVI siècle (15401600), Bd. 2: Sculpture - peinture - broderie - émail et faïence - orfèvrerie - armures, Paris 1986, S. 189f. Eine Beschreibung der ursprünglichen Dekoration in: Henri SAUVAL, Histoire et recherches des antiquités de la ville de Paris, Paris 1724, ND 1974, Bd. 2, S. 119, Bd. 3, S. 10. Vgl. Charles SAMARAN, Le Primatice et les Guises, d'après des documents inédits, in: Études italiennes 3 (1921) S. 129-136, 187-200. 
terre et melioracions qu'il a faictes en icelluy. Daneben mußten Maurer, Tischler, Schlosser und andere Handwerker bezahlt werden, die fast ununterbrochen an den Gebäuden beschäftigt waren ${ }^{10}$. Die Einrichtung des Hauses spiegelte den Stand seiner Eigentümer wider und verdeutlichte dem Besucher Macht und Reichtum der Guise, vor allem die kostbaren, die Wände der Säle schmückenden Tapisserien. Rund hundertfüfzig Stück, sowohl nach façon de Flandres als auch nach façon de Turquie hergestellt, zeigten farbige Blumenund Vogelmuster oder historische Szenen, wie die Geschichten Alexanders des Großen oder die des Merowingerkönigs Chlodwig. Der Vergleich mit Inventaren des in unmittelbarer Năhe gelegenen Hôtel der Montmorency zeigt, daß die Vorliebe der Guise für prächtige Teppiche, wertvolle Möbel, vor allem Betten, Gemälde und kostbare Leuchter, durch die ihr Hôtel zu einer würdigen Fürstenbehausung wurde, von anderen Adelsfamilien geteilt wurde ${ }^{11}$.

Einen weiteren Kauf tätigten Anna und François gemeinsam. Gegen Ende des Jahres 1555 erwarben sie die Grafschaft Nanteuil, was neben den Rechten an den Einkünften aus den Ländereien den Besitz des Schlosses, eines kleinen Waldes, der Gärten und Weinberge bedeutete ${ }^{12}$. Das Schloß, errichtet zu Zeiten Franz' I., war ein kleines Juwel und scheint nach dem Tod seiner einstigen Besitzerin Marguerite de Broye, die dort auch nach dem Verkauf ein lebenslanges Wohnrecht behalten hatte, zu einer der Lieblingsresidenzen der Herzogin von Guise (und fortan Gräfin von Nanteuil) geworden zu sein. Fast dreitausend Livres kosteten Anna in einem Jahr die Bauarbeiten an den Gebäuden, in ihrem Rechnungsbuch findet sich gar ein eigener, mit "Bastimens que Madame faict faire a Nantheuil« betitelter Abschnitt. Daneben versuchte sie, das Anwesen durch den Kauf d'une maison, jardin et fosses à poisson, avec demy arpent de terre zu vergrößern, und sie baute ein Pail-Mail-Spiel, sehr zur Freude ihrer Kinder. Mühe und Sorgfalt der Prinzessin scheinen Früchte getragen zu haben. In einem an Jacques de Savoie gerichteten und kurz nach seiner Hochzeit mit der Witwe des Herzogs von Guise verfaßten Brief wird das Schloß als la belle mayson de Madame votre femme bezeichnet. Später ging Nanteuil in den Besitz von Annas ältesten Sohn über, der es 1578 ver-

${ }^{10}$ Rechnungsbuch 1563 von Anna d'Este und ihren Kindern, in: BnF, Fr. 22433, fol. 31-118, hier fol. 79v, 82r. Das Zitat nach LANGLOIS, Les hôtels, S. 29.

1 Post-mortem-Inventar von François de Lorraine, 25. Febr. (1564), in: AST (Cast.), Int., PGN, cat. 5, m. 4, n. 3; Inventare des Hôtel de Montmorency, 1556, 1568, in: Léon MiRoT, L'hôtel et les collections du connétable de Montmorency, in: Bibliothèque de l'École des chartes 79 (1918) S. 311-413; 80 (1919) S. 152-229. Zu den Tapisserien: LANGLOIS, Les hôtels, S. 48-53.

${ }^{12}$ Eine Kopie des Kaufvertrags vom 11. Nov. 1555 ist aufgeführt in: Inventaire général des titres, fol. 23v. Die Transaktion wurde am 10. Jan. 1556 in die registres des insinuations du châtelet de Paris eingetragen, in: AN, Y 99, fol. 345v. Vgl. Acquisition du comté de Nanteuil, 5. Dez. 1555, in: BnF, Fr. 8182, fol. 91-99. 
kaufte. Heute ist, von einigen wenigen Überresten abgesehen, nichts von dem Schloß erhalten ${ }^{13}$.

Ihr Interesse an standesgemäßen, dem Geschmack der Zeit entgegenkommenden Gebäuden hatte Anna schon früh bekundet, und so überwachte sie die Arbeiten am Hôtel de Guise persönlich. Als molto bella hatte sie das gemeinhin als »Grand Ferrare« bekannte Schloß ihres Onkels Ippolito in Fontainebleau bezeichnet und ihn gebeten, ihr das Haus nach seinem Tod zu vermachen; tatsächlich sollte Ippolito das Schloß einige Jahre später seinem Schwager François schenken ${ }^{14}$. Auch der Kardinal von Lothringen scheint das Urteil der Prinzessin in Fragen der Kunst und Architektur geschätzt zu haben. In einem seiner Briefe forderte er sie auf, nach Paris zu kommen, affin de vous faire veoir les maisons que j'ay là autour, et celle que j'en ay nouvellement acquise, et que je vous en mette moy mesmes en possession. Das Schloß in Meudon scheint dem Kardinal besonders ans Herz gewachsen zu sein. Seine Schwägerin unterrichtete er über Details der Dekoration, und immer war er an Annas Meinung interessiert und an ihrer Zustimmung zu seinen Plänen: $S$ 'il $y$ a faulte, reprochez le moy ${ }^{15}$.

Die Aufgaben: patrimoine und maison

Ihr neuer Titel der Herzogin von Guise bedeutete für die Prinzessin zwar eine Steigerung des Prestiges und einen weitaus größeren Aufwand bei der Verwaltung des Familienbesitzes, finanziell waren sie und ihr Gemahl jedoch kaum bessergestellt. Während das Einkommen einiger der großen französischen Adelsfamilien zumindest vor Beginn der Religionskriege stieg, klagte Anna schon Anfang der 1560er Jahre: nous sommes si an arrières et devons tant que ye ne puis panser à rien, und François, der sich wiederholt zu Geldanleihen

${ }^{13}$ Rechnungsbuch 1563, fol. 98v; Rechnungsbuch 1567-1568 für die Grafschaft Nanteuil, in: AST (Piave), ACP, art. 806, par. 2, reg. 17, fol. 34r; Henri de Lorraine an François de Lorraine, 27. Apr. (1557), Villers-Cotterets, in: Joseph de CROZE, Les Guises, les Valois et Philippe II, Bd. 1, Paris 1866, S. 334; Florimond Robertet an Jacques de Savoie, 29. Juli 1566, Paris, in: BnF, Fr. 3211, fol. 104r. Zum Schloß Nanteuil-le-Haudoin: JeanPierre Babelon, Châteaux de France au siècle de la Renaissance, Paris 1989, S. 720.

${ }^{14}$ Francesco Primaticcio an François de Lorraine, 28. Okt. 1555, in: SAMARAN, Le Primatice, S. 193; Giulio Alvarotti an Ercole d'Este, 6. Sept. 1551, Fontainebleau, in: OcCHIPINTI (Hg.), Carteggio, S. 269; Anna d'Este an dens., 24. Nov. (1549), Paris, in: ASM, Canc. duc., Cart. prin. est. 1568 A/14. Der Hinweis auf die Schenkungsurkunde vom 30. Jan. 1556, in: BRIÉLE (Hg.), Collection de documents, S. 201. Eine Beschreibung des Grand Ferrare: Giulio BERTONI, Ippolito II d'Este, cardinale di Ferrara, in: Rivista storica italiana 41 (1924) S. 349-366, hier S. 352f.

${ }^{15}$ Charles de Lorraine an Anna d'Este, 28. Dez. 1552, Compiègne, in: BnF, Fr. 3118, fol. 44r u. LORRAINE, Lettres, S. 165. 
und Verpfändungen gezwungen sah, hinterlie $B$ der Gattin bei seinem Tod einen enormen Schuldenberg ${ }^{16}$. Vor allem aber brachte der Titel fur die Prinzessin neue organisatorische Aufgaben mit sich, denn die Oberaufsicht uber die Verwaltung der Güter überließ der Herzog ihr. Unterstützt wurde sie dabei von den Verwaltern des Familienbesitzes, die dem Paar jedes Jahr in dicken Büchern Rechenschaft über Einkünfte und Ausgaben ablegten, von Antoinette de Bourbon und von ihrem Schwager, dem Kardinal ${ }^{17}$.

Der größte und aufwendigste Teil von Annas neuen Aufgaben bestand in der Arbeit mit verschiedenen Papieren, mit Besitztiteln, Pachtverträgen und anderen Urkunden. Wie vielfaltig die verwalterische Tătigkeit der Prinzessin war, die sich von der Verpachtung ihrer Ländereien bis zur Vergabe von Ämtern erstreckte, kann aus dem Beispiel Provins ersehen werden. Die Herzogin war Herrin dieses südőstlich von Paris gelegenen Ortes, und die Memoiren des dortigen Priesters Claude Haton erzählen von den unterschiedlichsten Dingen, die es für sie zu erledigen gab, und den Bitten, mit denen sich die Bewohner an sie wandten. Eine dieser Bitten betraf den Abzug der Soldaten, die in Provins Quartier bezogen hatten, woraufhin Anna die Stadtherren zunächst gefragt haben soll, warum sie die Truppen überhaupt in die Stadt gelassen hätten und wozu ihnen ihre Mauern dienten, et très bien se mocqua d'eux. Doch dann setzte sie sich bei Karl IX. für die Erfullung dieses Wunsches ein und erreichte den Abzug der Soldaten ${ }^{18}$.

In welchem Maße dieser neue Tätigkeitsbereich der jungen Frau auch an die Führung eines landwirtschaftlichen Betriebes erinnern konnte, ist aus einem ihrer Briefe an die Herzogin von Nevers zu ersehen, in dem Anna die Kälber ihrer Zucht anpreist: Si les veaus que me demandiés estoyent [en] vos estables, votre bestial s'en montreroyt plus beau, car ce sont de plus grosses bestes que

${ }^{16}$ Anna d'Este an Renée de France, o.D. (ca. 1562), in: BnF, Fr. 3230, fol. 74r. Zur Entwicklung adligen Einkommens vor und während der Religionskriege: J. Russell MAJOR, Noble Income, Inflation, and the Wars of Religion in France, in: American Historical Review 86 (1981) S. 21-48.

${ }^{17}$ Charles de Lorraine an Anna d'Este, 28. Dez. 1552, Compiègne, in: LoRRAINE, Lettres, S. 165; Renée de Lorraine an François de Lorraine, o.D., in: BnF, Clair. 345, S. 281. Zu den Rechnungsbüchern vgl. die drei Bănde "Comte [...] de la terre et seigneurie de Guise que rend Jean Gaignebien, recepveur ordinaire du duché de Guise, à très excellente princesse et dame Madame Anne d'Est, duchesse de Guise«, 1562, 1563, 1565, in: MC, c.t., sér. D, reg. 14-16.

${ }^{18}$ Claude Haton, Mémoires (1553-1582), Bd. 1: Années 1553-1565, hg. v. Laurent BouRQUIN, Paris 2001, S. 456 (1563). Vgl. auch die Urkunden über die Emennung von Pierre Nauyer zum controlleur antien du grenier à sel du Provins durch Anna d'Este, März-Juni 1587, in: BnF, NAF 7974, fol. 111-116. Zu den anderen Aufgaben der Verwaltung: Jean d'Humières an François de Lorraine, o.D. (1550er Jahre), Compiègne, in: Kristen B. NEUSCHEL, Word of Honor. Interpreting Noble Culture in Sixteenth-Century France, Ithaca, London 1989, S. 69. 
j'ai james veu. Ein Blick auf die Tătigkeiten anderer Damen bestätigt nicht nur den landwirtschaftlichen Charakter, sondern auch die breitgefächerten Aufgaben, die bei der Verwaltung eines großen Besitzes für die Mutter oder Gemahlin des Herm anfielen. So kümmerte sich Jeanne de Gontault als Witwe im Namen ihres minderjährigen Sohnes um die Geldgeschăfte, um Kăufe und Verkäufe von Land, sie verhandelte mit Pächtem und pflegte die Beziehungen zu den Klienten ihres verstorbenen Gatten, alles Dinge, die es furr Anna schon zu Lebzeiten des Herzogs zu erledigen galt. Das Beispiel der Isabelle de Forz verdeutlicht darüber hinaus, welchen Profit die Fürstinnen, zumindest in Zeiten guter wirtschaftlicher Konjunktur, durch flexibles Handeln aus ihrem Grund und Boden erwirtschaften konnten ${ }^{19}$.

In Zeiten des Friedens kümmerten sich François und seine Gemahlin gemeinsam um ihre Angelegenheiten, wenn der Herzog jedoch vollständig vom Dienst für den König beansprucht war, zeichnete Anna allein verantwortlich ${ }^{20}$. Dabei stand sie stets in engem Kontakt mit ihrer Schwiegermutter, welche die Verwaltung von Joinville leitete. Eines der von Antoinette an die Herzogin von Guise gerichteten Schreiben, in dem es unter anderem um Pachtzahlungen geht, verdeutlicht die Vielfaltigkeit der zu erfüllenden Pflichten. Der Brief ist nicht datiert, seine Sprache läßt aber vermuten, daß er in der ersten Hălfte der 1550er Jahre geschrieben wurde, als Antoinette die Schwiegertochter in die Geschäfte um den Familienbesitz einwies. Genau erklärt sie darin der Jüngeren, was zu tun sei, läßt aber auch eigene Entscheidungen und Verantwortlichkeiten zu: S'ilz vyennent vers vous pour vous en faire demande, vous en respondrez comme vous vouldrez. Schon als Joinville zum Fürstentum erhoben werden sollte, hatte die Prinzessin fur ihre Schwiegermutter die notwendigen Papiere zusammengesucht, und später übersandte sie an Antoinette die für Reparaturen an den Brücken und Mauern von Joinville bestimmten Zahlungsanweisungen des Königs, eine Handlung, die sie in ihrem Begleitschreiben als mon debuoir en labsence de mons' mon mary bezeichnete ${ }^{21}$.

${ }^{19}$ Mavis MATE, Profit and Productivity on the Estates of Isabella de Forz (1260-92), in: Economic History Review 33 (1980) S. 326-334. Das Zitat: Anna d'Este an Marguerite de Bourbon, o.D. (1550er Jahre), in: BORDEAUX, Les amants, S. 48. Das Wort in Klammern wurde von mir anhand BnF, Fr. 4711, fol. 64r korrigiert. Zu Jeanne de Gontault: KALAS, Widow's Place. Zum landwirtschaftlichen Charakter der Aufgaben vgl. auch den Brief von Anne Baillet an Jeanne Luillier, in: Barbara B. DIEFENDORF, Widowhood and Remarriage in Sixteenth-Century Paris, in: Journal of Family History 7 (1982) S. 379-395, hier S. 388.

${ }^{20} \mathrm{~F}$. de La Chaussé an François de Lorraine, 7. Apr. 1553, Joinville, in: BnF, Clair. 346, fol. 255r; Charles de Lorraine an Ercole d'Este, 9. Jan. 1554, Fontainebleau, in: Lettres Charles, S. 184.

${ }^{21}$ Antoinette de Bourbon an Anna d'Este, 13. Mai o.J., in: BnF, Fr. 22437, fol. 25r; Anna d'Este an Antoinette de Bourbon, (5.) Febr. (1551), 24. Apr. (1560?), in: PIMODAN, Antoinette de Bourbon, S. 336, 351 f., Zitat S. 352. 
Außer für den Familienbesitz hatte die Herzogin von Guise für ihren eigenen Haushalt und die in ihren Diensten stehenden Personen zu sorgen. Elf Frauen und über zwanzig Männer waren 1552 für sie tätig, eine Zahl, die sich in den folgenden Jahren noch vergrößern sollte. Zum Zeitpunkt des Todes ihres ersten Gemahls wurde Anna von einem mehr als fünfzig Personen zählenden Gefolge aufgewartet, dem sie Löhne in Höhe von über viertausend Livres bezahlte. Während die meisten männlichen Mitglieder des Hofes relativ klar umrissenen Tätigkeiten nachgingen, teilten die Damen den Alltag ihrer Herrin, begleiteten sie zu Festen und Turnieren, assistierten bei repräsentativen Handlungen und erledigten täglich anfallende Aufgaben wie Nähen und Servieren $^{22}$. Einige der dames und demoiselles verblieben nur zeitweilig in Annas Diensten, andere begleiteten sie über die Jahre hinweg, wie etwa Jeanne de Jumelles, ihre Ehrendame. Jeanne war spătestens seit 1552 bei der italienischen Prinzessin beschäftigt, auf deren Gehaltsliste ihr Name lange zu finden ist, selbst noch nach Annas Heirat mit dem Herzog von Nemours. Auch die demoiselles Vallère und Cassandre sowie die femme de chambre Jacqueline blieben der Herzogin viele Jahre treu. Ein beständiger Wechsel ist vor allem bei den Italienerinnen zu verzeichnen. So findet sich Anfang der 1550er Jahre noch die aus Ferrara stammende Margherita Brasavola in Annas Diensten, eine Kindheitsgefährtin, die als fille du corps an Renées Hof gemeinsam mit der Prinzessin erzogen worden war und diese dann nach Frankreich begleitet hatte. Vier Jahre später ist sie durch die demoiselle Francisque ersetzt, deren Name sicher die französische Form der italienischen Francesca darstellt. Laura Montina trat 1559 zum letzten Mal als demoiselle in Erscheinung; im September, nachdem Anna sie an einen Italiener verheiratet hatte, kehrte auch sie nach Ferrara zurück. Aus Lauras Empfehlungsschreiben ist die Aufmerksamkeit zu erkennen, welche die Herzogin den ihr zur Erziehung anvertrauten Mädchen zukommen ließ:

Monseygneur, la Laure que y'ai norrie et marriée au seigneur Antonio Galleazzo Bentivoglio s'en retourne à Ferrare. Ye ne l'ay point voullu laysser partir sens l'acconpagner de sette lettre, pour vous suplier très humblement, Monseygneur, l'avoyr pour reccoumandée. Car ye vous asseure qu'elle le merite, pour estre autant sage et honneste qu'il est possible, et est bien estimée telle en sette conpagnie et bien fort regretée de Monseigneur mon mari et de moy ${ }^{23}$.

${ }^{22}$ Haushalt von Anna d'Este und François de Lorraine, 1552-1561, in: BnF, Fr. 22429, fol. 130-165, Fr. 22437, fol. 42-68; Haushalt von Anna d'Este, 14. Okt. 1563, in: BnF, Fr. 22429, fol. 166f., Fr. 22437, fol. 69-71, Clair. 816, S. 179-183. Vgl. Jacqueline BouCHER, Le cardinal de Lorraine, premier ministre de fait ou d'ambition (1559-1574), in: Yvonne BELlENGER ( $\mathrm{Hg}$.), Le mécénat et l'influence des Guise. Actes du Colloque de Joinville 1994, Paris 1997, S. 295-310, hier S. 306; MÜNSTER, Funktionen, S. 344, 353 f.

${ }^{23}$ Anna d'Este an Ercole d'Este, 23. Sept. 1559, Reims, in: ASM, Canc. duc., Cart. prin. est. 1568 A14. Zu Margherita Brasavola: Rechnungsbiucher 1540, fol. 50r, 1544, fol. 80v. Zu 
Ähnliche Beobachtungen wie bei den weiblichen können bei den männlichen Angestellten gemacht werden. Neben ständig wechselnden Namen finden sich die des Schneiders Pierre Paullet, des Stallmeisters Jean-Baptiste und des Kammerdieners Luc Mabileau, die von Beginn an bis zum Tod des Herzogs, einige sogar darüber hinaus, in Annas Diensten standen. Jean Beguyn, der als Reitknecht in den herzoglichen Ställen begonnen hatte, war 1560 zum maître de litière aufgestiegen, verantwortlich für die Wartung von Annas Sänften, während die jahrelang für die Verwaltung von Brot, Wein und Geschirr zuständigen Herren 1563 als Pensionäre verzeichnet sind. Der wichtige Posten des Finanzverwalters und Sekretärs war bis zum Jahre 1560 von Nicolas de Portille, hinter dessen französischer Namensform sich wahrscheinlich ein Spanier verbirgt, bekleidet. Die genannten Namen machen es deutlich: Italiener sind unter den männlichen Bediensteten der Prinzessin, anders als es bei den weiblichen der Fall ist, kaum zu finden. Allein hinter dem Namen des Almosenpflegers Bauturnus verbirgt sich der aus Brescia stammende Boturnio Boturnei, und auch bei ihrem Stallmeister Jean-Baptiste könnte es sich um einen ihrer Landsmänner handeln, womit Anna der im französischen Adel zu beobachtenden Modeerscheinung nachgekommen wäre, einem Italiener die Leitung der Ställe zu überlassen ${ }^{24}$.

Bei der Organisation ihres Haushaltes scheint die junge Herzogin von Guise einiges Talent bewiesen zu haben. In einem Ende des Jahres 1550 verfaßten Brief an den Schwiegervater berichtet François, seine Gemahlin habe très bien commencée à entendre le faict du mesnaige de ceste [...] maison. Die an ihre weiblichen Angestellten ausgezahlten Löhne liegen zwar unterhalb dessen, was etwa die Königin von Navarra ihren dames und demoiselles bezahlen konnte, doch ist bei den von Anna während der 1550er Jahre gewährten Gehältern ein deutlicher Anstieg zu verzeichnen, der in den Lohnlisten von Jeanne d'Albret nicht zu finden ist ${ }^{25}$. Daß die Prinzessin darüber hinaus gut für

den Schwierigkeiten der Identifizierung weiblicher Mitglieder der maison: Ruth KLENMAN, Social Dynamics at the French Court: The Household of Anne of Austria, in: French Historical Studies 16 (1990) S. 517-535, hier S. 524-526. Zu Stellenwechseln von Angestellten adliger Haushalte: Sharon KETTERING, The Household Service of Early Modern French Noblewomen, in: French Historical Studies 20 (1997) S. 55-85, hier S. 83.

${ }^{24}$ Zu Pierre Paullet: Post-mortem François. Zu der Mode, italienische Stallmeister einzustellen: Jean-François DUBOST, La France italienne, XVI'-XVII siècle, Paris 1997, S. 199, der Jean-Baptiste ebenfalls für einen Italiener hält: Ibid. S. 403. Annas späterer Kutscher hieß "Tasse«, was die Übersetzung des italienischen "Tasso « sein dürfte: Rechnungsbücher 1591, 1599-1606 von Anna d'Este, in: AST (Piave), ACP, Art. 806, par. 2, reg. 170 u. AST (Castello), Int., PGN, cat. 5, m. 11, n. 1; Vertrag über den Kauf von Kleidungsstücken zwischen Anna d'Este und Jean Berault, 20. Febr. 1602, in: AN, m.c. VIII, 559, fol. 188v.

${ }^{25}$ François de Lorraine an Ercole d'Este, 23. Dez. (1550), Blois, in: ASM, Canc. duc., Cart. prin. est. 1568/13; Rechnungsbücher von Jeanne d'Albret, 1556, 1565, in: RAYMOND, Comptes, Bd. 10, S. 566f., Bd. 11, S. 120f. Vgl. die Tabelle in: EURICH, Power, S. 110. Zum 
ihre Bediensteten zu sorgen wußte, geht neben dem zitierten Empfehlungsschreiben für ihr Ehrenfräulein Laura aus den Eintragungen in ihren Rechnungsbüchern hervor. Nicht anders als Jeanne d'Albret, die ihre Angestellten ebenfalls mit großzügigen Extrazuwendungen bedachte, beschenkte auch Anna die Mitglieder ihres Haushaltes. Ihr Portemanteau Mathurin erhielt mehr als sieben Livres, eine Summe, die sein monatliches Einkommen bei weitem überstieg, und Jacques, einem ihrer Lakaien, ließ sie im selben Jahr achtunddreißig Livres auszahlen, pour apprendre ung mestier. $\mathrm{Da}$ die Herzogin auch Mitgiften für diejenigen übernahm, welche andernfalls nicht hätten heiraten können, ist daran zu erkennen, daß sie der Amme eines ihrer Söhne zweihundertfünfzig Livres als Hochzeitsgeschenk übermachte, die in Raten an den Gatten ausgezahlt wurden ${ }^{26}$.

\section{Die Schwierigkeiten: Frankreich im Krieg}

Der Grund, weshalb Anna von Beginn an Verantwortung zu übernehmen hatte, sich um die Verwaltung des Familienbesitzes kümmern und selbst wichtige Entscheidungen treffen mußte, lag zu einem großen Teil an der fast ständigen Abwesenheit ihres Gemahls. Ununterbrochen war François vom königlichen Dienst in Anspruch genommen, sowohl in Friedenszeiten als erst recht in Zeiten des Krieges. Die wichtigste Aufgabe der Fürstin in Kriegszeiten kann als "holding the fort» bezeichnet werden, da sie, nicht anders als eine mittelalterliche Burgherrin, es dem Herzog dank ihrer Sorge um den Familienbesitz überhaupt erst ermöglichte, in die Schlacht zu ziehen. Der Krieg stellte daher für die italienische Prinzessin eine janusköpfige Angelegenheit dar. Im Falle einer siegreich bestandenen Schlacht ihres Gatten, später der Söhne, hatte auch sie Anteil an deren Ruhm, erhielt Glückwünsche und Geschenke, wie etwa aus einem Brief des Königs bezüglich eines militärischen Erfolgs ihres ältesten Sohnes hervorgeht: il peust aporter tant d'heur et a vous et a tout ce qui vous touche. In der Regel brachte der Krieg aber weitaus mehr Schrecken als Ehre mit sich ${ }^{27}$.

Einkommen von Hausangestellten: Cissie FAIRCHILDS, Domestic Enemies. Servants and Their Masters in Old Regime France, Baltimore, London 1984, S. 54-58.

${ }^{26}$ Rechnungsbuch 1563, fol. 78v, 93r. Das Einkommen Mathurins im Jahre 1563 betrug 60 1.t., das Jacques' 50 l.t., das der Amme wohl 40 1.t. Zu den von Jeanne d'Albret gewährten Extrazuwendungen vgl. ihr Rechnungsbuch des Jahres 1556, in: RAYMOND, Comptes, Bd. 10, S. 573f.

${ }^{27}$ Heinrich III. an Anna d'Este, ca. 3. Mai 1577, Chenonceaux, in: HENRI III, Roi de France, Lettres, Bd. 3, hg. v. Pietre ChampION, Michel FranÇOIS, Paris 1972, S. 237. Zur Rolle von Fürstinnen im Krieg: Kristen B. NEUSCHEL, Noblewomen and War in Sixteenth-Century France, in: Michael WOLFE (Hg.), Changing Identities in Early Modern France, Durham, 
Ho conforto nissuno, erzählte Anna dem Vater, nachdem François Ende des Jahres 1556 mit seinem Heer nach Italien aufgebrochen war, per esser cosi addolloratta. Dem bereits zitierten Schreiben an die Herzogin von Nevers fugte die Prinzessin, nachdem sie die Kälber ihrer Zucht erwähnt hatte, den Wunsch bei, que tout le malheur de la guerre tombast sur eux [et sur de leur semblable] et que nos amis fussent hors de danger, und der Herzogin von Montmorency schrieb sie, als sich ihre Männer gemeinsam auf dem Schlachtfeld befanden: quant ye panse, qu'il[s] sont touryours an danger, ye ne me puis guièrre reyouir ${ }^{28}$. Wie oft sie um das Leben ihres Gemahls bangen mußte, geht aus zahlreichen Briefstellen hervor. Im Juli 1557 etwa schrieb der Kardinal von Lothringen dem noch in Italien weilenden François, seine Gattin sei ung peu jaulne, da sie von aller Welt gehört habe, ihr Mann sei verloren. Nachdem Antoine de Bourbon während der Belagerung von Rouen verletzt worden war, beruhigte die Königinmutter ihre Freundin, dem Herzog von Guise gehe es gut, obwohl er sich in unmittelbarer Nahe des Königs von Navarra aufgehalten habe. Und als Anna schließlich Nachricht von der Schlacht vor Dreux erhielt, war sie wie leblos vor lauter Sorge, erholte sich jedoch, als sie hörte, daß der Herzog wohlauf $\operatorname{sei}^{29}$.

Welche Auswirkungen ein militärischer Sieg ihres Gemahls auf das Leben der Prinzessin haben konnte, zeigt die Eroberung von Calais. Die als uneinnehmbar geltende Stadt war Anfang des Jahres 1558 von François und seinen Truppen gesturmt worden - ein Sieg, der doppelt galt, wurde er von den Zeitgenossen doch als Wiedergutmachung der verheerenden Niederlage von Saint Quentin gewertet. Entsprechend enthusiastisch waren die Reaktionen: Es gäbe keinen Leutnant des Königs, der von französischem und ausländischem Kriegsvolk so geliebt und verehrt würde wie ihr Gatte, schrieb Louis seiner

London 1997, S. 124-144; Penny RICHARDS, The Guise women: Politics, war and peace, in: Jessica MUNNS, DIES., Gender, Power and Privilege in Early Modern Europe, Harlow 2003, S. 159-170; Éliane VIENNOT, Les femmes dans les "troubles« du XVI siècle, in: Catherine MARAND-FouQUET (Hg.), Guerres civiles, Toulouse 1997, S. 79-96. Zu den Aufgaben einer mittelalterlichen Burgherrin: Mary ERLER, Maryanne KOWALESKI, Einleitung zu: DIES. (Hg.), Women and Power in the Middle Ages, Athens, London 1988, S. 1-17, hier S. 5. Die Formulierung »holding the fort« in: FINLEY-CROSWHITE, Engendering the Wars, S. 133.

${ }^{28}$ Anna d'Este an Ercole d'Este, 19. Dez. 1556, Grenoble, in: ASM, Canc. duc., Cart. prin. est. 1458/22; Dies. an Marguerite de Bourbon, o.D. (1550er Jahre), in: BORDEAUX, Les amants, S. 48. Die Worter in Klammern wurden von mir anhand BnF, Fr. 4711, fol. 64r ergănzt. Dies. an Madeleine de Savoie (?), Herzogin von Montmorency, o.D., in: BnF, Fr. 3554, fol. 35r. Da der Brief kein Datum trägt, kann nicht mit Sicherheit bestimmt werden, ob er nicht an Diane de France, Gemahlin von François de Montmorency, gerichtet ist, wahrscheinlich kann er aber auf den Sommer oder Herbst 1562 datiert werden.

${ }^{29}$ Charles de Lorraine an François de Lorraine, 6. Juli 1557, Compiègne, in: LoRRAINE, Lettres, S. 270; Katharina von Medici an Anna d'Este, (15. Okt. 1562), in: MÉDICIS, Lettres, Bd. 1, S. 420; Thomas Smith an den Rat der Königin Elisabeth I., 22. Dez. 1562, SaintDenis, in: Calendar Elizabeth, 1867, S. 585. 
Schwägerin. Als klug Rechnender fügte er den Nutzen hinzu, den die Guise aus diesem Sieg zu ziehen gedachten, denn Charles de Lorraine wollte den König bitten, dem Bruder als Anerkennung für seine treuen Kriegsdienste die Grafschaft Oye zu überlassen ${ }^{30}$. Der Kardinal von Lothringen schenkte der Herzogin aus lauter Freude eine wertvolle Perlenkette, und sein Kollege aus Ferrara schrieb der Nichte:

se ben ho sempre creduto, che l'amor suo verso Mons. ${ }^{r}$ Ill. ${ }^{\text {mo }}$ nostro di Guisia fosse in colmo, si come era debito verso un cosi honorato, et valoroso marito, voglio nondimeno tener per fermo, che ci sara stato anche luogo per accrescerlo in haverlo veduto uscire con si felice essito d'una impresa tanto difficile, et tanto importante il che son certo, che quanto piu porta di riputatione et di gloria a S. Ex. ${ }^{\text {tia }}$ tanto piu risulta in allegrezza, et contento a lei ${ }^{31}$.

Rund zwanzig Jahre später sollte Annas ältester Sohn in der Schlacht von Dormans zwar siegen, dabei aber eine Verletzung davontragen, die ihn die Hälfte der linken Wange und einen Teil des Ohres kostete, so daß auch er, nicht anders als sein Vater, den Beinamen Le Balafré erhielt. In vielen der an die Mutter des Herzogs gerichteten Briefe verschwindet die lebensbedrohliche Verletzung jedoch unter den enthusiastischen Bekrättigungen der Ehre, die durch diesen Sieg errungen worden sei. So schrieb einer der Gratulanten: lo[u]é soyt dieu que le malade et blessé nous dem[e]ure avec ung très grant onneur, il ne sera plus si beau, mes [mais] pasiance, und Luigi d'Este ließ die Schwester wissen, daß ihr Sohn zwar un poco ferito sei, die Narbe aber una bella marca abgeben würde und jeden, der ihm in Zukunft ins Gesicht blicke, an diesen relevatissimo servitio für die Krone erinnern müsse. Die einzige, die sich ernsthafte Sorgen um die Mutter des Verletzten zu machen schien, war Katharina von Medici $^{32}$.

In Zeiten des Krieges war ein Brief, manchmal ein kurzer Besuch die einzige Möglichkeit für das Herzogspaar, miteinander zu kommunizieren. Als François sich Ende 1552 mit seiner Armee in Metz verschanzt hatte und von

${ }^{30}$ Louis de Lorraine an Anna d'Este, o.D. (Jan. 1558), in: BnF, Fr. 3231, fol. 85v. Die Grafschaft Oye war von François de Lorraine und seinen Truppen wăhrend der Eroberung von Calais eingenommen worden. Am Ende erhielt der Herzog vom König 250000 l.t. und ein Haus in Calais: Rechnungsbuch 1563, fol. 54r; RoMER, Origines, Bd. 2, S. 220, Anm. 1. Vgl. die Liste der im Zusammenhang mit der Eroberung von Calais entstandenen Lobgedichte auf den Herzog, in: David J. HARTLEY, Les poètes français et la prise de Calais (janvier 1558), in: Bibliothèque d'Humanisme et Renaissance 56 (1994) S. 719-728.

${ }^{31}$ Ippolito d'Este an Anna d'Este, 1. Febr. 1558, Ferrara, in: MONCH (Hg.), Denkwürdigkeiten, S. 100. Zum Geschenk der Perlenkette: ROMIER, Origines, Bd. 2, S. 220.

${ }^{32}$ Ein Unbekannter an Anna d'Este, o.D. (Okt. 1575), in: BnF, Fr. 3347, fol. 71r; Luigi d'Este an dies., 12. Okt. 1575, Paris, in: Fr. 3423, fol. 66r; Katharina von Medici an Jacques de Savoie, 14. Okt. 1575, Blois, in: MÉDICIS, Lettres, Bd. 5, S. 155. Vgl. Heinrich III. an Anna d'Este, 18. Okt. 1575, in: HENRI III, Lettres, Bd. 2, S. 278f.; Louis de Lorraine an dies., o.D. (Ende 1575), in: PolZAT, Princesse de Clèves, S. 144. 
den Truppen Karls V. belagert wurde, übernahm der im nahegelegenen Verdun stationierte Maréchal de Saint-André die Rolle des Boten. Der Marschall wisse immer eine Möglichkeit, ihre Briefe an den Gemahl weiterzuleiten, versuchte Charles seine Schwägerin zu trösten, die in großer Sorge um den Herzog war $^{33}$. Anna, ihre Schwiegermutter sowie deren Töchter Renée und Antoinette, bemuht, dem Gatten, Sohn und Bruder so nahe wie möglich zu sein, hatten sich nach Reims und dann nach Joinville zurückgezogen, wo sie die Überbringer seiner Schreiben empfingen. Einfach war es für Saint-André nicht, den Kontakt mit dem belagerten Metz aufrecht zu erhalten, doch er wußte, welche Bedeutung ein Brief, selbst eine kurze Nachricht für die Herzogin haben konnte, und so versprach er, ihr sofort einen Boten zu schicken, sobald er Neuigkeiten von François hätte, saichant bien que pour le presant je ne vous sçauroys fere service plus agreable ${ }^{34}$.

Andere Damen baten Anna regelmäßig um Neuigkeiten vom Kriegsgeschehen, wie es etwa die Königin von Navarra in Zeiten tat, in denen sich ihr Gemahl gemeinsam mit François auf dem Schlachtfeld befand. Als Gegenleistung bot Jeanne d'Albret der Prinzessin Hilfe an für den Fall, daß sie vom Krieg unmittelbar betroffen wäre: Falls sie belagert werden sollten, so die Königin, solle Anna ihr Nachricht zukommen lassen, afin que je vous mayne secours $^{35}$. Wie begründet derartige Vorkehrungen waren und wie groß die Gefahr auch furr die Herzogin und ihre Kinder, in das Kriegsgeschehen verwickelt zu werden, kann an dem Gerücht abgelesen werden, das im September 1562 bis nach England gelangte und demzufolge die Gemahlin, die Mutter und der älteste Sohn des Herzogs von Guise François d'Andelot in die Hände gefallen seien. Denn ganz so galant, wie Brantôme es beschreibt, waren die Zusammentreffen am Rande des Kriegsgetümmels nicht. Der Autor berichtet von einem Gespräch des späteren Heinrich IV. mit Anna und ihrer Tochter Catherine kurz vor der Schlacht von Dreux, und er schließt seinen Bericht mit den Worten: En telles belles et gentilles parolles, quasy en forme de dialogue, se passarent les devis de ce grand roy et de ces belles princesses ${ }^{36}$.

Erleichterung brachte die Zeit, in der François vom Dienst für den König beurlaubt war, es gar zu einem Frieden kam, und er sich zu seiner Familie

${ }^{33}$ Charles de Lorraine an Anna d'Este, 28. Dez. 1552, Compiègne, in: LORRAINE, Lettres, S. 165.

${ }^{34}$ Jacques d'Albon an Anna d'Este, 29. Dez. (1552), Verdun, in: BnF, Fr. 3200, fol. 117r.

${ }^{35}$ Jeanne d'Albret an Anna d'Este, o.D. (1554), (1557), in: BOURBON, ALBRET, Lettres, S. 89, 149. Vgl. auch die Information über das Wohlergehen des Konnetabel, in: Anna d'Este an Madeleine de Savoie, 7. Juli 1562, in: MC, c.l., sér. L, vol. XVIII, fol. 165r.

${ }^{36}$ Pierre de Bourdeilles, abbé et seigneur de BRANTÓME, Euvres complètes, hg. v. Prosper MÉrimÉE, Louis LACOUR, Bd. 5, Paris 1876, ND 1977, S. 114. Das Gerücht über die Gefangennahme der Familie des Herzogs: Thomas Randolph an William Cecil, 18. Sept. 1562, Spyney, in: Calendar Elizabeth, 1867, S. 305. 
begeben konnte. Während einer Kampagne gegen die Engländer im Herbst 1549 gab Heinrich dem Herzog von Aumale die Gelegenheit, sich einen ganzen Monat zu seiner schwangeren Gemahlin zuruckzuziehen, woruber sich selbst Ippolito d'Este in Rom erfreut zeigte, pour le plaisir et contentement que je sçay que vous et elle en receverez. Und als François mit seinem Heer aus Italien zurückkehrte, erhielt er vom Kardinal von Lothringen den Rat, sich so schnell wie möglich zu seiner Gattin zu begeben, die ihm wohl kaum die Hälfte ihres Bettes verweigern würde. Or, madame, si nous avons la pais, deliberez vous d'estre belle et bien en point et passons le tans le demeurant de noz jours, schrieb der Kardinal seiner Schwägerin und bezeugte damit den gemeinsamen Wunsch nach Frieden. Der einzige, der seinen Platz eher auf dem Schlachtfeld als am heimischen Herd sah, war der Herzog selbst. Leicht gelangweilt und enttäuscht, qu'il ne s'offre chose par deçà [...] que de mesnage et femme grosse, teilte François Ende 1558 dem Konnetabel Montmorency mit, er wolle sich, nachdem er zu Hause seine Pflicht erfüllt habe, zurück zum König begeben, um so bald wie möglich seinen Dienst wieder aufzunehmen ${ }^{37}$.

2. »Dieu ne nous veult laisser sans heritier« Die Mutter

Zwischen ihrem achtzehnten und ihrem vierzigsten Lebensjahr sollte Anna d'Este zehn Kinder zur Welt bringen, sieben als Herzogin von Guise, weitere drei wahhrend ihrer Ehe mit Jacques de Savoie. Nie sonst in den Quellen tritt die Prinzessin in ihrer Korperlichkeit so klar hervor, wie in den Monaten ihrer Schwangerschaften. In ihren Briefen sprechen sowohl Anna als auch die jeweiligen Korrespondenzpartner von bizarren Eßgelüsten, Fehlgeburten und ausbleibenden Regelblutungen. Nie wurde der Körper der Herzogin so genau beobachtet wie in Zeiten, in denen sie sich in besonderen Umständen befand, nie seine Veränderungen so präzise wahrgenommen und beschrieben. Über den gesundheitlichen Zustand und die medizinische Versorgung ihrer Kinder kann hingegen kaum etwas gesagt werden, da Krankenjournale und Rezept-

${ }^{37}$ Ippolito d'Este an François de Lorraine, 29. Sept. 1549, Rom, in: BnF, Fr. 20534, fol. 105r; Charles de Lorraine an dens., 19. Okt. 1557, in: LORRAINE, Lettres, S. 287; Ders. an Anna d'Este, 1. Sept. (1558), in: Ibid. S. 334; François de Lorraine an Anne de Montmorency, o.D. (Ende 1558), in: BoULLÉ, Histoire, Bd. 1, S. 503f. 
sammlungen, wie sie adlige Mütter zwei Jahrhunderte später für ihren Nachwuchs anlegen sollten, in den Archiven nicht zu finden sind ${ }^{38}$.

\section{Schwangerschaften, Geburten und Taufen}

Die erste Schwangerschaft der Prinzessin war ein großes Ereignis, mit Spannung erwartet von der Familie, vor allem aber von ihrem Gemahl, welcher sich einen, besser mehrere männliche Nachkommen erhoffte. Fünf Monate nach ihrer Hochzeit schrieb Anna dem Vater:

Io pigliarò l'ardire di scrivere a V.E. forsi una bugia, che è ch'io mi dubito d'esser gravida, ma non ne sono anchora ben certa. Io mi sento molto male il doppoi disnare e vomito qualche volta il doppoi disnare e pure non lascio d'ingrassarmi. Et il maggiore segno che ì in questo è ch'io ho fallito di quindici giorni le mie purgationi. Io non ne ho ditto niente a Messer Nicolò [den Überbringer des Briefes] perchè non ne sono ben certa 39 .

Bereits wenige Tage später konnte die Nachricht bestätigt werden, sehr zur Zufriedenheit der Guise. Die Glückwünsche ließen nicht lange auf sich warten, und der Kardinal von Ferrara gab seiner Hoffnung Ausdruck, seine Nichte möge zur Freude der beiden Familien von einem bello figliuolo maschio entbunden werden. Alle weiteren Schwangerschaften der Herzogin wurden nicht minder eifrig verbreitet und enthusiastisch begrüßt. Seine Frau fuhle sich schlecht, und wahrscheinlich würde es ihr erst in neun Monaten besser gehen, meldete François im Juli 1553 nach Ferrara, und als Anna ihr viertes Kind erwartete und die Nachfolge gesichert schien, schrieb Charles de Lorraine der Schwester in Schottland: Dieu ne nous veult laisser sans heritier, qui est le plus grand heur qu'il nous peust envoyer ${ }^{40}$.

Über das Befinden der werdenden Mutter war man in Ferrara stets gut unterrichtet. Im dritten Monat ihrer ersten Schwangerschaft teilte Anna dem Vater mit, daß sie in den vergangenen Wochen nicht zu-, sondern abgenommen habe, sie nicht essen könne und es ihr schlecht ginge, daß aber alle ihr versichert hätten, sie würde sich besser fühlen, sobald sie ihr Kind zum ersten Mal

${ }^{38}$ Claudia KollBACH, Karoline Luise von Baden-Durlach als Mutter ihrer kranken Kinder. Medizinische Praktiken als Teil der Prinzenerziehung in der zweiten Hälfte des 18. Jahrhunderts, in: Zeitenblicke 4 (2005), Nr. 3, unter: www.dipp.zeitenblicke.de/2005/3/Kollbach/index html.

${ }^{39}$ Anna d'Este an Ercole d'Este, 28. Mai 1549, in: ASM, Canc. duc., Cart. prin. est. $1568 \mathrm{~A} / 14$.

${ }^{40}$ Ippolito d'Este an Anna d'Este, 26. Juli 1549, Rom, in: ASM, Casa 153; François de Lorraine an Ercole d'Este, 8. Juli (1553), in: ASM, Canc. duc., Cart. prin. est. 1568/13; Charles de Lorraine an Marie de Lorraine, 29. Dez. 1554, Saint-Germain-en-Laye, in: LORRAINE, Lettres, S. 189. 
gespürt habe ${ }^{41}$. Wie bei anderen Fürstinnen der Zeit waren auch Annas Angehörige über kaum etwas besser informiert als uber die Bewegungen des Kindes im Mutterleib. Nie sei es ihr besser gegangen, als nachdem sie ihr Ungeborenes sich bewegen gefuhlt habe, schrieb die Prinzessin einige Monate später, und auch während der folgenden Schwangerschaften sind ihre Briefe voll von Mitteilungen darüber, ob sie ihr Kind gespurt habe oder nicht. Noch anläßlich der letzten Entbindung der mittlerweile Vierzigjährigen wurde dem besorgten Gatten versichert, daß sich das Ungeborene bewegt habe und die Mutter gesund sei $^{42}$. Der psychologische Effekt darf hierbei nicht unterschätzt werden, schließlich waren die Bewegungen des Ungeborenen ein wichtiger Indikator dafür, daß das Kind lebte, denn selbst wenn sich die Prinzessin nur un pocho male fuhlte, hatte sie paura grande vor einer Fehlgeburt. Neben den Bewegungen stellte auch das Wachsen des Ungeborenen ein gutes Zeichen dar und wurde nicht weniger genau beobachtet. So berichtete Alvarotti von einem seiner Besuche bei der Herzogin von Aumale, sie habe einen derart dicken Bauch, daß sich darin entweder zwei Kinder befinden müßten oder ein riesengroßes $^{43}$.

Die Geburt des ersten Kindes war monatelang vorbereitet worden. Dem Kardinal von Ferrara war es eine Ehre, sein Haus in Fontainebleau für dieses Ereignis zur Verfügung zu stellen: Et si Notre Seigneur me rend si heureux que ce puisse estre d'ung filz masle, j'en aymeray mon logis beaucop davantaige. Stets sahen sich die Besitzer der Schlösser, die für die Entbindungen der Herzogin ausgewählt wurden, geschmeichelt und waren bemüht, das neue Familienmitglied mit allem nur möglichen Prunk zu empfangen: Sua Signoria reverendissima fa un bellissimo aparato, e farà una gran spesa, né vol che monsignor di Gissa vi spenda un soldo, hieß es etwa anläßlich der dritten Entbindung der Herzogin ${ }^{44}$. Die Anweisungen, welche Anna rund drei Jahrzehnte später anläßlich der Geburt des ersten Kindes ihres Sohnes Charles erteilen sollte, lassen den bei ihren eigenen Niederkünften getriebenen Aufwand erahnen. Mit den kostbarsten Tapisserien sollten die Wände des Rau-

41 Anna d'Este an Ercole d'Este, 15. Juni (1549), in: ASM, Canc. duc., Cart. prin. est. $1568 \mathrm{~A} / 14$.

${ }^{42}$ Anna d'Este an Ercole d'Este, 1. Nov. 1549, Paris, in: ASM, Canc. duc., Cart. prin. est. 1568 A 14 ; Renée de France an Jacques de Savoie, 8. Mai 1572, Montargis, in: MONCH (Hg.), Denkwürdigkeiten, S. 197. Über Wachsen und Bewegung ihres Kindes berichtet auch Margarete von Navarra in einem Brief an Franz I. aus dem Jahre 1543, in: STEPHENSON, Power, S. 143.

${ }^{43}$ Anna d'Este an Ercole d'Este, 24. Nov. (1549), Paris, in: ASM, Canc. duc., Cart. prin. est. 1568 A/14; Giulio Alvarotti an dens., 30. Nov. 1549, Paris, in: OCCHIPINTI (Hg.), Carteggio, S. 239.

44 Ippolito d'Este an François de Lorraine, 29. Sept. 1549, Rom, in: BnF, Fr. 20534, fol. 105r; Tommaso Zerbinato an Ercole d'Este, 19. Nov. 1553, Paris, in: OCCHIPINTI (Hg.), Carteggio, S. 301. Die Rede ist vom Schloß des Kardinals von Lothringen in Meudon. 
mes, in dem der Säugling das Licht der Welt erblicken würde, bespannt, das Bett aber mit goldenem Damast bedeckt werden. So wird auch die Behauptung glaubhaft, die Herzogin habe mitunter bis zu 20000 Scudi für die Vorbereitungen ihrer Entbindungen ausgegeben ${ }^{45}$.

Während der gesamte Hof im Dezember 1549 die Niederkunft der Herzogin von Aumale in Fontainebleau erwartete, konnte der werdende Vater selbst nicht anwesend sein, da er im Dienste des Königs unterwegs war. Am letzten Tag des Jahres, abends um acht Uhr und dreiundfünfzig Minuten, gebar Anna schließlich ihr erstes Kind, einen Sohn ${ }^{46}$. Schon wenige Tage später bekam der Gesandte des Herzogs von Ferrara das Neugeborene zu sehen. Alvarotti war beeindruckt, sowohl von dem Baby als auch von den ihm zu Ehren getätigten Ausgaben. Den Knaben, so schreibt er, habe er tutto nudo zu sehen bekommen, e l'ho trovato grande e grosso e bellissimo. Hanno fatto una grandissima spesa di letti e paramenti molto ricchi. Anna war glücklich. Der kürzlich von einem Kind entbundenen Herzogin von Nevers wünschte sie, einen ebenso schönen Sohn zu haben wie sie selbst, mais non pas si gros, und voller Stolz auf ihre neue Erfahrung fugte sie hinzu, sie hätte ihr gerne als Hebamme gedient. François konnte seine Freude, als er in Fontainebleau eintraf und endlich seinen Sohn in den Armen halten durfte, kaum bändigen. Dem Schwiegervater teilte er mit: Il fault que je fasse le fou père de vous faire entendre l'ayze que j'ay receue de ce qu'il a pleu à Dieu me donner ung fils ${ }^{47}$.

Alle weiteren Niederklinfte der Prinzessin wurden nicht minder interessiert verfolgt und kommentiert. Allein anläßlich der Geburt von Catherine, einzige Tochter aus der Ehe mit dem Herzog von Guise, wußten manche Gratulanten

\footnotetext{
${ }^{45}$ Anweisungen von Anna d'Este zur Einrichtung der Răume für die Niederkunft von Henriette de Savoie, Herzogin von Mayenne, o.D. (1578), in: BouILLÉ, Histoire, Bd.3, S. 502504. Die genannte Summe in: Boturnio Boturnei (an Renée de France), o.D. (vor 1555), in: FONTANA, Renata di Francia, Bd. 3, S. XLII.

${ }^{46} \mathrm{Vgl}$. die mit »Henrici Ducis Guisiae nativitas anno 1549 die 31 . Decembr. hora $8.53^{\mathrm{m}}$ post meridiem « überschriebene Darstellung der Planetenkonstellation zum Zeitpunkt der Geburt, in: BnF, Dup. 844, fol. 61v. Zur Abwesenheit des Vaters: François de Lorraine an Ercole d'Este, 3. Nov. 1549, 20. Jan. (1550), Paris, Fontainebleau, in: ASM, Canc. duc., Cart. prin. est. 1568/13; Costanzo de' Preti an Katharina von Österreich, Herzogin von Mantua, 28. Nov. 1549, Paris, in: ASMan, AG 642, fol. 507r. Im Januar 1550 bezahlte Renée de France 36 Livres an einen Lakaien, der ihr Nachricht von der Niederkunft ihrer Tochter brachte: Emmanuel RODOCANACHI, Une protectrice de la réforme en Italie et en France: Renée de France, duchesse de Ferrare, Paris 1896, ND 1970, S. 188, Anm. 2.

${ }^{47}$ Giulio Alvarotti an Ercole d'Este, 2. Jan. 1550, Melun, in: OCCHIPINTI (Hg.), Carteggio, S. 240; Anna d'Este an Marguerite de Bourbon, o.D., in: BordEAUX, Les amants, S. 48; François de Lorraine an Ercole d'Este, 20. Jan. (1550), Fontainebleau, in: ASM, Canc. duc., Cart. prin. est. 1568/13. Ebenso erfahren zeigte sich Anna, als die Gattin ihres Bruders schwanger zu sein schien: Anna d'Este an Alfonso d'Este, 29. März 1560, Amboise, in: Ibid. Nr. 1568 A/14: Si Madame ma seur est grosse mandes-le-moy et ye vous feray faire toutte les drogues en Franse qui seront esperimantée et mieus faittes.
} 
ihre Enttäuschung nicht zu verbergen. Anna selbst hatte auf einen Knaben gehofft, ebenso die Königin, deren Namen das Mädchen dann tragen sollte, doch es kam anders. Er hătte ihnen zwar eher einen Sohn als eine Tochter gewünscht, mais vous avez l'ung et l'aultre, versuchte Gaspard de Coligny zu trösten, und als guter Vater schrieb der Herzog von Ferrara seiner Ältesten: Se ben harei desiderato che V.S. havesse più presto fatto un altro bel puttino, nondimeno essendo piaciuto a Dio ch'ella habbia partorito una figliola con sua salute, io mi contento del tutto ${ }^{48}$.

Die Taufen der Kinder, vor allem natülich des Erstgeborenen, waren keine minder bedeutende Angelegenheit als die Entbindungen. Für Henri wurden im Hof des Grand Ferrare kostspielige Aufbauten errichtet, und der König, der dem Knaben seinen Namen gab, sollte ihn persönlich uber das Taufbecken halten. Zwei Tage und zwei Nächte wurde gefeiert, wurden Bankette, Maskeraden und Kampfspiele veranstaltet. Für einen unterhaltsamen Zwischenfall sorgte der Säugling selbst: Quando tenevano il figliuolo sopra la fonte lui pissò in una manica del giupon de Sua Maestà, la quale dicendo rel pissa, el pissa!c, si entrò in una risata in quella sala la magiore del mondo ${ }^{49}$. Die Entscheidungen für die jeweiligen Taufpaten und Namensgeber der Kinder spiegeln politische Situationen und Allianzen wider, zeigen dabei immer aber die Macht der Guise. Während der älteste Sohn und die Tochter zu Beginn der 1550er Jahre die Namen des regierenden Königspaares erhielten, wurde der 1557 geborene Antoine von zwei bewährten Kriegsgefährten seines Vaters uber das Taufbecken gehalten: Antoine de Bourbon, König von Navarra, und Jacques d'Albon, Maréchal de Saint-André. Annas siebtes Kind kam hingegen zu einer Zeit auf die Welt, in der die politischen Allianzen in Frankreich schon lange nicht mehr klar waren. Obwohl François auf dem Schlachtfeld weiterhin die Sache seines Königs vertrat, und dieser das Kind sogar über das Taufbekken hielt, wurde der jüngste Sohn nicht mehr nach einem Mitglied des französischen Adels benannt, sondern erhielt den Namen des späteren Kaisers Maximilian $^{50}$.

${ }^{48}$ Gaspard de Coligny an François de Lorraine, 25. Juli 1551, Chalon-sur-Saône, in: BnF, Fr. 20461, S. 151; Ercole d'Este an Anna d'Este, 11. Aug. 1551, Ferrara, in: BnF, Clair. 344, fol. 208r. Vgl. auch die weniger enttäuschten Stimmen: Diane de Poitiers an Antoinette de Bourbon, (25.) Juli (1551), Blois, in: PoYTIERS, Lettres, S. 88f.; Charles de Cossé an François de Lorraine, 4. Aug. 1551, in: BnF, Clair. 344, fol. 196r.

${ }^{49}$ Giulio Alvarotti an Ercole d'Este, 4. Febr. 1550, Melun, in: OCCHIPINTI (Hg.), Carteggio, S. 243.

${ }^{50}$ Vgl. die mit »Extraict de l'aage des enfans de feu Monsieur de Guise« betitelte Aufzăhlung der Kinder, in: BnF, Fr. 4741, fol. 138f. Zu den Taufen: Nicolas de Lorraine an François de Lorraine, 5. Juli 1551, in: BnF, Fr. 20467, S. 35; Ercole d'Este an Anna d'Este, 8. Mai 1554, in: BnF, Fr. 3121, fol. 39r; Charles de Lorraine an Francois de Lorraine, 3. Mai 1557, in: LORRAINE, Lettres, S. 263; Eintrag vom 22. Nov. 1562, in: Journal de ce qui 


\section{Strategien der Karriereplanung für den Nachwuchs}

Nachdem sie zehn eigene Kinder großgezogen hatte, von denen das letzte noch funf Jahre jünger war als ihre Schwiegertochter Anna, sollte sich Antoinette de Bourbon mit großer Energie der Erziehung ihrer Enkel widmen. Nicht nur die Kinder der Herzogin von Guise galt es zu versorgen, auch der Nachwuchs ihres Sohnes Claude, Herzog von Aumale, der mit einer Tochter von Diane de Poitiers vermăhlt war, wuchs in Joinville auf. $\mathrm{Zu}$ diesen Knaben und Mädchen sollten sich später noch die Sprößlinge ihres jüngsten Sohnes René gesellen. Die Herzoginwitwe war glücklich, tout mon petit peuple, wie sie sich ausdrückte, um sich zu haben. Schon bald wollte sich Antoinette nicht mehr von ihren Enkelkindern trennen. Als Anna und François den Wunsch äußerten, ihren Sohn zu sich nach Paris zu holen, erhielten sie Besuch vom Kardinal von Lothringen, der ihnen klar machte, die Zeit, den Knaben der Obhut seiner Großmutter zu entziehen, sei noch nicht gekommen: à quoy ilz veullent obeyr et ont changé l'oppinion qu'ilz en avoyent prinse, de sorte qu'il ne s'en parlera plus pour cest heure ${ }^{51}$. Hatten die Kleinen ein bestimmtes Alter erreicht, übernahmen entweder Hauslehrer ihre Erziehung, sie kamen in ein collège oder sie wurden der Obhut von Verwandten übergeben. Mit acht oder neun Jahren begann der älteste Sohn seinem Vater zu folgen, wăhrend die jüngeren Knaben oftmals gemeinsam mit einem der größeren Brüder erzogen wurden ${ }^{52}$. Catherine wuchs bei ihrer Tante Renée, Äbtissin des Klosters Saint-Pierre in Reims auf. Ihre Aufgabe bestand darin, eines Tages Macht und Ansehen der Guise durch eine glanzvolle Heirat zu vergrößern, und der immer um das Wohl seines Hauses bemüte Kardinal von Lothringen hatte schon vor ihrer Geburt die Fäden zu spinnen begonnen. Als das Mädchen dann auf der Welt war, schrieb er dem Bruder: Il est vray que j'eusse bien desiré un fils mais j'espere que vous recommancerez de sy bonne heure que bien tost vous amanderez la faulte, et ceste fille, sy Dieu plaist, nous fera une bonne alience ${ }^{53}$. In den folgenden Jahren wurde das Thema von Catherines Hochzeit immer wieder aufgenommen. Für ihre Familie stellte das Mädchen ein wichtiges Kapital dar, welches eingetauscht werden konnte gegen Ämter und Würden, oder zur

s'est passé en France durant l'année 1562, principalement dans Paris et à la cour, in: Revue rétrospective 5 (1834) S. 81-116, 168-212, hier S. 199f.

${ }^{51}$ Antoinette de Bourbon an Marie de Lorraine, 29. Sept. 1553, in: LORRAINE, Balcarres, S. 169; Charles de Lorraine an Antoinette de Bourbon, 21. Apr. (1553), Saint-Germain-enLaye, in: LORRAINE, Lettres, S. 171.

52 Rechnungsbuch 1563: Ausgaben für Charles' precepteur (fol. 66r), für Catherines pension et habillemens (fol. 77r) und für Maximilien, depuis qu'il a esté à Joinville avec [seinem älteren Bruder] François (fol. 67r).

${ }^{53}$ Charles de Lorraine an François de Lorraine, 26. Juli 1551, Blois, in: LORRAINE, Lettres, S. 154. 
Absicherung derselben genutzt wurde. Auch die Herrscher bedienten sich der Sprößlinge ihrer Fürsten, um Verträge zu garantieren oder Frieden zwischen verfeindeten Parteien zu stiften. So forderte Katharina von Medici den Herzog von Guise auf, seine zehnjährige Tochter an den Hof zu schicken mit der Erlaubnis, sie a modo suo verheiraten zu dürfen, ohne daß er sich in die Verhandlungen einmische. Auf die Nachfrage des Vaters antwortete sie, das Mädchen solle dem Sohn des Prinzen von Condé, einem der größten Gegner der Guise, versprochen werden, per meglio compor questi tumulti ${ }^{54} . \mathrm{Zu}$ einem Abschluß kam jedoch keine der vielfältigen Verhandlungen, und Catherine heiratete erst viele Jahre nach dem Tod des Vaters, als ihre Mutter bereits Herzogin von Nemours war.

Doch nicht nur die Tochter war dazu bestimmt, schon früh im Namen der Familie eine Verbindung einzugehen, auch für den fünfjährigen Sohn Charles, ou celuy de leurs autres enffans qui premier sera trouvè en aage nubille, unterzeichneten Anna und François ein Versprechen. Der älteste Sohn blieb aus den Verhandlungen ausgeschlossen; seine Hand sollte vergeben werden, sobald sich eine dem Machtstreben der Guise entgegenkommende Gelegenheit bot. Die versprochene Heirat zwischen Charles, dem zukünftigen Herzog von Mayenne, und Renée d'Anjou läßt tief blicken, steht sie doch für das Interesse der Guise am Königreich Neapel, und obwohl auch dieses Heiratsprojekt nie verwirklicht werden sollte, verdeutlicht es die hochtrabenden Pläne, welche das Herzogspaar hinsichtlich ihrer nicht für die kirchliche Laufbahn bestimmten Kinder hegte ${ }^{35}$.

Annas fünfter Sohn, François, war gemeinsam mit seinem älteren Bruder Louis für die Kirche vorgesehen. An seiner Biographie kann exemplarisch die Erziehung eines der jüngeren, in der Hierarchie der Geschwister zweitrangig plazierten Kindes demonstriert werden. So wie Charles im Schatten des erstgeborenen Henri stand, rangierte François stets hinter seinem Bruder Louis, der schon früh mit einem Teil der Pfründe seines Onkels ausgestattet worden war. Der am letzten Tag des Jahres 1559 geborene François verbrachte die ersten sechs Lebensjahre bei der Großmutter in Joinville. Der Obhut seines Onkels übergeben kam der Knabe dann in das vom Kardinal von Lothringen in Reims gegründete Collège Saint-Patrice, pour estre instruit tant ez bonnes

\footnotetext{
${ }^{54}$ Avviso aus Frankreich, o.D. (Ende Apr. 1562), in: ASF, MP 4850, fol. 111r. Zu den anderen Heiratsplänen: François de Lorraine an Ercole d'Este, 12. Febr. (1554), Paris, in: ASM, Canc. duc., Cart. prin. est., 1568/13; Ders. an Alfonso d'Este, 18. März (1558), Fontainebleau, in: Ibid.; Anna d'Este an Ercole d'Este, 18. März (1558), Fontainebleau, in: Ibid. Nr. $1568 \mathrm{~A} / 14$; Avis de la cour, 29. Sept. 1559, in: BnF, Fr. 20624, fol. 55r: François de Lorraine soll des Amt des grand chambellan an den Herzog von Longueville abtreten, der dafür seine Tochter heiraten soll. Einen vorlaufigen Heiratsvertrag hatten die Guise im Januar 1558 aufsetzen lassen: BRIÈLE (Hg.), Collection de documents, S. 203.

${ }^{55} \mathrm{Vgl}$. den Hinweis auf das Heiratsversprechen vom 6. Mai 1559, in: AN, Y 107, fol. 54r.
} 
mours qu'ez arts et sciences, dont son âge et son bel esprit le rendirent capable. Er wurde Kanonikus an der Kathedrale Notre-Dame in Reims und assistierte seinem Onkel, dem Bischof, bei der Messe. Im collège erlernte er die Künste der Grammatik und Rhetorik, machte dabei erstaunliche Fortschritte und sprach bald nur noch auf Latein. Dann geschah etwas Außergewöhnliches: Anstatt sich mit dem erworbenen Wissen zufriedenzugeben und auf ein zukünftiges Leben als Abt eines reichen Klosters vorzubereiten, wandte sich François an die Professoren seines collège und bat in einer wohlformulierten lateinischen Rede darum, die Kunst der Logik studieren zu dürfen. Die von den rhetorischen Fähigkeiten des Dreizehnjährigen und der Ungewöhnlichkeit seiner Bitte gleichermaßen beeindruckten Zuhörer gestatteten ihm die Fortführung seiner Ausbildung. Sicher hätte François in seinen Studien erstaunliche Ergebnisse erzielt, doch sollte er deren Beginn nie erleben, da er Ende Oktober 1573 starb. Charles de Lorraine nahm sich den Tod seines Lieblingsneffen, dem er während dessen Krankheit keinen Augenblick von der Seite gewichen war, sehr zu Herzen. Kein Wunder: Vierzig Stunden lang hatte der Junge vor Schmerzen geschrien, ohne daß ihm geholfen werden konnte. Seiner Schwägerin schrieb der Kardinal: Je ne suis pas bon à vous consoler car je cuide estre le plus ennuyé du munde car j'ay extrememant perdu, und er schloß seinen Brief mit den Worten: Mes larmes me font abreger ${ }^{56}$.

\section{„Monsieur le prince«: Die Erziehung eines Erstgeborenen}

Unangefochtener Mittelpunkt der Geschwisterschar und Liebling der Großmutter, von den Erwachsenen mit Aufmerksamkeit beobachtet, umhegt und gepflegt wie kein anderer, tritt Henri de Lorraine, der den Titel »Prinz von Joinville« trug, als Kind deutlicher in den Quellen hervor als seine Brüder. In Briefen und Berichten findet sich sein Name häufiger erwähnt, seine Person betreffende Tatbestände wurden sorgfältiger vermerkt, und mit dem Tod des Vaters bekam er seinen eigenen Haushalt und mithin eine eigene Buchführung. Auf ihn als erstgeborenen Sohn war das Interesse der Familie gerichtet, besonders während der ersten Lebensjahre, die nicht selten kritisch für das Überleben eines Kindes waren. Der Herzog und die Herzogin von Ferrara erkundigten sich ständig nach ihrem Enkel, Antoinette bezeichnete ihn als

${ }^{56}$ De la vie très-exemplaire et très-heureuse mort de François de Lorraine, in: OUDIN, Histoire, 5800, fol. 60-62, Zitat fol. 60r; Charles de Lorraine an Anna d'Este, 24. Okt. (1573), Reims, in: LORRAINE, Lettres, S. 659. Eine von François' Lehrer verfaßte Vita des Knaben in: Nicolas BouchER, La Conjonction des Lettres et des Armes des deux tresillustres Princes Lorrains, Charles Cardinal de Lorraine Archevesque et Duc de Rheims, et François Duc de Guyse, Reims 1579, fol. 76-85. 
notre petit mignon, und dem Vater wurde wăhrend seiner Abwesenheit der Sohn als le plus beau prince qu'il est posible de voir beschrieben ${ }^{57}$. Privilegiert waren auch die im Dienste des Knaben stehenden Personen, wie seine Amme Catherine Mirebel, die gemeinsam mit der Nährmutter des Herzogs von Guise bis zu dessen Tod auf Annas Gehaltsliste stehen sollte. Sie war eine der vier Ammen, die von Antoinette in Joinville gespeist wurden und konnte, im Gegensatz zu vielen anderen Hausangestellten, schreiben. Später sollte sie auch Annas jüngsten Sohn aus erster Ehe nähren, und noch im Herbst 1593, mehr als vierzig Jahre nach Henris Geburt, erhielt sie von ihrer ehemaligen Brotgeberin ein großzügiges Geldgeschenk ${ }^{58}$.

Ständiger Begleiter des Prinzen Henri war sein gleichnamiger, nur wenig älterer Cousin, der erstgeborene Sohn des Herzogs von Aumale. Die Knaben verbrachten ihre Kindheit zusammen, erst bei der Großmutter in Joinville, dann im Gefolge ihrer Văter. Kinder gemeinsam zu erziehen war eine gängige Praxis französischer Adelsfamilien, und nicht selten wurden die zukünftigen Spielgefährten gleich nach ihrer Geburt einander bestimmt: Puisqu'il a plu au seigneur Dieu me faire tant de bien que de m'avoir donné ung fils, ce sera pour estre compaignon du vostre, comme nous avons esté estans jeunes et petits, schlug etwa der König von Navarra seinem Waffengefährten vor, und Anna d'Este wünschte sich, ihre Tochter wäre einige Jahre älter, um gemeinsam mit ihrer Cousine Maria Stuart erzogen werden zu können ${ }^{59}$. Die Entscheidung für oder gegen einen zukünftigen Kindheits- und Jugendgefährten wurde von den Eltern getroffen. So sind aus einem Schreiben der Herzogin von Guise, mit dem sie einen Knaben aus dem Gefolge ihres Sohnes für einige Zeit der Obhut des Bruders übergab, ihre Gründe für die Wahl dieses Spielgefăhrten zu erfahren: $y$ 'aime bien fort le père et désire, après qu'il arra eu set honneur que d'estre norri de votre mayn, le retirre[r] auprès de mondit fils.

${ }^{57}$ Antoinette de Bourbon an Ercole d'Este, 30. Jan. (1550), Fontainebleau, in: ASM, Canc. duc., Cart. prin. est. 1568/13; (Florimond) de Biencourt an François de Lorraine, 2. Juni (1550), Joinville, in: BnF, Fr. 20543, fol. 135r. Vgl. die Briefe von Ercole d'Este und Renée de France an François de Lorraine, 1550-1552, in: BnF, Clair. 344.

${ }^{58}$ Catherine erhielt eine jährliche Bezahlung von 40 1.t.: Haushalte von Anna d'Este, 15521563, in: BnF, Fr. 22429. Zur Speisung in Joinville vgl. die Auflistung vom 1. Sept. 1552, in: BouILlÉ, Histoire, Bd. 1, S. 550. Im Juli 1566 unterzeichnete Catherine für den Kammerdiener des Herzogs von Guise eine Empfangsbestätigung: Inventaire des bagues que Madame de Nemours a donné et donne à Monseigneur le duc de Guyse son filz, 22. Juli 1584, in: AST (Cast.), Int., PGN, 5. cat., m. 6, n. 6. Zur Nährung des jüngsten Kindes vgl. das Kapitel »La despence de Maximilian Monsieur«, in: Rechnungsbuch 1563, fol. 67v. Zu dem Geldgeschenk: Rechnungsbuch 1593-II (Sept.).

${ }^{59}$ Antoine de Bourbon an François de Lorraine, 22. Sept. 1551, Coucy, in: Alphonse de RuBLE, Antoine de Bourbon et Jeannne d'Albret, Bd. 1, Paris 1881, S. 334. Der besagte Sohn sollte früh sterben, es handelt sich also nicht um den späteren Heinrich IV. Anna d'Este an Marie de Lorraine, 1552, in: LORRAINE, Balcarres, S. 110. 
Der Wunsch, dem Vater des Jungen, einem der serviteurs de notre mayson, einen Gefallen zu tun, spielte dabei nicht minder eine Rolle als die Hoffnung, das Kind später als ebenso treuen Anhänger im Umfeld ihres Sohnes wiederzufinden. Tatsăchlich sollte sich Anna dann nach Jahren bei Alfonso erkundigen, ob der Jüngling bereit sei, ihrem Ältesten in seiner grand escuyrye zu dienen $^{60}$.

Hielt Henri sich bei Hofe auf, spielte er mit den Söhnen und Töchtern der Fürsten und mit den Kindern des Königs. Die erheblich ältere Diane de France gab sich mit ihm ab, nicht anders als die spätere spanische Königin Elisabeth von Valois oder deren jüngere Schwester Margarete. Der Sohn des Prinzen von La Roche-sur-Yon zählte ebenso zu seinen Kindheitsgefăhrten wie der spătere Heinrich III., über den der venezianische Botschafter zu berichten wußte, der Prinz von Joinville sei il suo più caro gewesen, und selbst der König vergnügte sich mit Henri und dessen Freunden bei einem Fangspiel ${ }^{61}$. Das Verhältnis des Prinzen zu seinen Geschwistern stellte sich distanziert dar, doch mit einem fast văterlichen Verantwortungsgefühl des Ältesten den jüngeren Brudern gegenuber: J'ayme bien mon frère Charles et mon frère Louis, car ils sont les plus jolis du monde, mais je ne sçais quand j'auroy veu mon petit frère, lequel j'aimerois mieux. Je serois leur gouverneur et leur apprendrois leur cour, heißt es in einem seiner Briefe über die Bruder, während er uber die Schwester lediglich zu sagen wußte: $j$ 'ay vu ma søur et ma cousine $d^{\prime}$ 'Aumale aussi qui sont bien saiges et bien jolies ${ }^{62}$.

Monsieur votre fyls se porte fort byen, schrieb der Konnetabel der besorgten Anna, als ihr noch nicht dreizehnjähriger Sohn gemeinsam mit dem Vater der Belagerung von Rouen beiwohnte ${ }^{63}$. Mochte sich die Mutter auch sorgen Henri war glücklich, denn seit seiner frühesten Kindheit wünschte er, es sei-

${ }^{60}$ Anna d'Este an Alfonso d'Este, o.D. (Anfang 1560er), 5. Apr. 1568, Montereau, Paris, in: ASM, Canc. duc., Cart. prin. est. 1568 A/14, 1458/22. Es handelt sich um den Sohn von Monsieur de Crêne, einem der maîtres d'hôtel des Königs, der im Haushalt des jungen Herzogs von Guise 1563 als superintendant des affaires verzeichnet ist: BnF, Clair. 816, S. 184.

${ }^{61}$ Henri de Lorraine an François de Lorraine, 2. Okt. 1557, Saint-Germain-en-Laye, in: BnF, Fr. 20467, fol. 157r. Zu den Spielgefährten: ValoIS, Mémoires, S. 73f.; Henri de Lorraine an François de Lorraine, (28. Dez. 1556), Saint-Germain-en-Laye, in: CROZE, Les Guises, Bd. 1, S. 331f. Croze datiert den Brief auf den 22. Jan. 1557, da er falschlicherweise annahm, der Herzog von Guise wäre erst Ende Dez. 1556 nach Italien aufgebrochen. Das Zitat: Michele Suriano an die Serenissima, 4. Nov. 1561, Paris, in: Michele SURIANO, Marc' Antonio BARBARO, Venetian Ambassadors at the Court of France, Despatches, 1560-1563, hg. v. Henry LAYARD, Lymington 1891, S. LVI.

${ }^{62}$ Henri de Lorraine an François de Lorraine, 27. Apr. (1557), Villers-Cotterets, in: CROZE, Les Guises, Bd. 1, S. 333f. Bei dem petit frère handelt es sich um Antoine, der kurz zuvor geboren worden war und den Henri noch nicht gesehen hatte. Mit der cousine d'Aumale ist Catherine gemeint, die älteste Tochter von Claude de Lorraine und Louise de Brézé.

${ }^{63}$ (Anne de) Montmorency an Anna d'Este, 14. Okt. 1562, Au camp devant Rouen, in: BnF, Fr. 3180, fol. 70r. 
nem Vater gleichzutun und ein großer Krieger zu werden. Als Siebenjähriger interessierte er sich für nichts so sehr wie für die Jagd, für Windhunde und Reitpferde. Sein Onkel, der den Prinzen sicher nur scherzhaft für ein der Kirche geweihtes Leben zu interessieren suchte, mußte eine herbe Niederlage einstecken:

J'ay ouy de beaux sermons que mon oncle a faicts à Reims, mais je vous promets que je ne les saurois raconter tout du long, car ils estoient si très-longs qu'il ne m'en souvient pas de la moitié. $l l$ [...] m'a demandé si je ne voulois pas estre chanoine à Reims, mais je lui respondis que j'aimerois mieux estre auprès de vous pour rompre une lance ou une espée sur quelque brave espagnol ou bourguignon, pour éprouver si j'ay bon bras, car $j$ 'aime mievx escrimer ou rompre lance que d'estre toujours enfermé dans une abbaye avec le froc ${ }^{64}$.

Entsprechend groß war die Begeisterung des Knaben für Pferde und das Reiten. Während einer Vorführung anläßlich der Hochzeit des Dauphin mit Maria Stuart kamen die beiden Henri, der von Guise und der von Aumale, auf hölzernen Pferdchen in den Saal geritten, cheminans et allans de telle sorte qu'on eust dict iceulx estre vivans, und wenige Monate später saßen sie wieder im Sattel, diesmal in einem richtigen, um den Truppen ihrer Väter einen Besuch abzustatten. Çà, çà, mes petits princes, çà, mettez pied à terre, wurden sie von Blaise de Monluc aufgefordert, der den Jungen, nachdem sie abgestiegen waren, die Piken über die Schulter hängte. Dann ließ er sie an der Spitze der Kompanie marschieren: Leurs gouverneurs estoient si aises, et tous nos capitaines, de veoir ces enfans marcher comme ils faisoient, qu'il n'y avoit nul qui $n$ 'en eust un bon presage ${ }^{65}$.

Auf die Charakterbildung des jungen Prinzen hat die Tatsache, ständig im Mittelpunkt zu stehen und alle Aufmerksamkeit auf sich gerichtet zu wissen, sicherlich Auswirkungen gehabt. Doch dies allein kann das schwierige Wesen des späteren Herzogs von Guise nicht erklären, schließlich genossen die erstgeborenen Söhne aller Adelsfamilien derartige Privilegien. Der gewaltsame Tod des geliebten Vaters wird seine Spuren im Gemüt des Dreizehnjährigen hinterlassen haben, und bestimmte Charakterzüge scheint der Prinz schon in frühen Kinderjahren aufgewiesen zu haben. On avoist dict à madame ma grand mère que j'estois opiniâtre, gestand er dem Vater, doch nur, um

\footnotetext{
${ }^{64}$ Henri de Lorraine an François de Lorraine, 27. Apr. (1557), Villers-Cotterets, in: CROZE, Les Guises, Bd. 1, S. 333.

${ }^{65}$ Discours du grand et magnifique triumphe faict au mariage de tresnoble et magnifique Prince François de Vallois Roy-Dauphin, filz aisné du tres-chrestien Roy de France Henry ij. du nom, et de treshaulte et vertueuse Princesse madame Marie d'Estrevart Roine d'Escosse (1558), in: L. CIMBER, Félix DANJOU (Hg.), Archives curieuses de l'histoire de France depuis Louis XI jusqu'à Louis XVIII, 1. Ser., Bd. 3, Paris 1835, S. 249-259, Zitat S. 257; Blaise de MonluC, Commentaires, 1521-1576, hg. v. Paul CoURTEAULT, Paris 1964, S. 454f. (Aug. 1558).
} 
sogleich zu beschwichtigen: Wäre er tatsächlich so halsstarrig wie behauptet, würde er von seinem Erzieher gehörig bestraft werden. Nicht immer ganz brav zu sein, dessen scheint sich Henri bewußt gewesen zu sein. Je suis devenu un peu bon, kommentierte der Knabe seine Anstrengungen und seine Hoffnung, bald ein guter Junge zu werden: J'espère me conduire si sagement, et luy [der Mutter] seray si obeissant, que vous et elle en auront contentement ${ }^{66}$.

Das Bild, welches schließlich Margarete von Valois in ihren Memoiren von dem neunjährigen Henri zeichnet, bestätigt den Eindruck eines schwierigen, latent aggressiven Kindes, das gewohnt war zu befehlen. Die Königin schreibt aus der Rückschau, wissend um das Schicksal des späteren Herzogs von Guise, so daß ihre Erzählung mit Vorsicht betrachtet werden muß. Übergangen werden soll sie aber dennoch nicht, schon allein deshalb, weil sie die Atmosphäre des Hofes schildert, in der Annas Kinder aufwuchsen. Schließlich hat Margaretes Biographin ihr die Fähigkeit attestiert, in den Memoiren mit ungewöhnlicher Präzision Orte, Szenen und Gewohnheiten zu rekapitulieren ${ }^{67}$. Margarete erinnert sich, wie ihr Vater, der König, sie eines Tages aufgefordert habe, einen der beiden im Raum anwesenden Knaben zu ihrem serviteur zu erwählen: Den Prinzen von Joinville oder den Marquis de Beaupréau, Henri de Bourbon. Das Mädchen entschied sich für letzteren, und auf die Frage des Vaters, warum sie den Dunkelhaarigen dem schöneren blonden Prinzen vorzöge, antwortete sie: Pour ce qu'il était plus sage, et que l'autre ne peut durer en sa patience qu'il ne fasse toujours mal à quelqu'un, et veut toujours être le maître ${ }^{68}$.

3. »Ci sono alcune donne che valgono per molti uomini« Die Politikerin

Erste Erfahrungen mit der Funktionsweise des französischen Hofes hatte Anna schon vor ihrer Abreise aus Ferrara sammeln können, denn ihre Integration in die politischen Vorgänge und der Versuch seitens des Hofes, sie in das System der Parteiungen einzubinden, begann bereits im Sommer 1548. Während der Herzog von Ferrara noch in Piemont weilte, hatte seine Tochter, entgegen aller

\footnotetext{
${ }^{66}$ Henri de Lorraine an François de Lorraine, 27. Apr. (1557), 26. Sept. o.J. (1557?), VillersCotterets, Paris, in: CrozE, Les Guises, Bd. 1, S. 334 u. BnF, Fr. 20467, S. 164.

${ }^{67}$ Éliane VIENNOT, Einleitung zu: VALOIS, Mémoires, S. 15-65, hier S. 40.

${ }^{68}$ VALOIS, Mémoires, S. 74. Die Episode spielt im Jahre 1559, Margarete war also sechs Jahre alt und nicht, wie sie selbst behauptet, vier bis fünf. $\mathrm{Zu}$ den vorangegangenen Ausführungen vgl. auch das Kapitel "L'enfance d'un prince«, in: Jean-Marie CONSTANT, Les Guise, Paris 1984, S. 52-57.
} 
Gebrăuche, die dem Brăutigam die Initiative einer ersten Kontaktaufnahme zuwiesen, ihrem zukünttigen Gemahl einen Brief geschrieben. Der uber diese Grenzüberschreitung des jungen Mädchens beunruhigte Ercole versuchte daraufhin, seinem Schwiegersohn die Situation zu erklären:

Penso che V.E., se ben forsi non le sarà dispiaciuto cognoscere il bono animo de la principessa mia figliola verso lei, haverà però trovato novo, sicome lo trovai anch'io, ch'ella, essendo figlia e nei maneggi che si trova con V.E., sia stata la prima a scriverle quelle quattro righe che l'haverà veduto. Ma voglio ch'ella sappia che essa mia figliola, subito ch'io fui gionto qua, mi narrò come fu constretta da Madama sua matre, ad instantia de Monsignor de Vandomo, a scriverle [...]. E dirò di più che 'l predetto Monsignor di Vandomo la pregò strettissimamente, gionta che fusse in corte, a volersi mostrar molto intrinseca con lui, e domesticarsi con la signora principessa di Navarra sua consorte [...]. E tutto questo di sopra ho voluto scriver a predetta V.E. in excusatione di mia figliola, a fine ch'ella non la judicasse più leggiera di quello che io spero in Dio ch'ella la trovarà in effetto ${ }^{69}$.

Tatsăchlich verbirgt sich hinter dieser Affäre der Versuch des späteren Königs von Navarra, der Heinrich nach Piemont begleitet und sich in diesem Rahmen anscheinend auch nach Ferrara begeben hatte, die Prinzessin von Beginn an der >richtigen، Gruppierung der Hofgesellschaft zuzuführen. Während Renée darauf aus war, ihre Tochter in das religiös tolerante Umfeld der Margarete von Navarra einzugliedern, ihr also der Vorstoß des Herzogs von Vendôme gelegen kam und sie ihn daher auch unterstützte, scheint sich Anna zumindest zu Beginn den Intrigen des Hofes ferngehalten zu haben. Ein halbes Jahr nach ihrer Ankunft in Frankreich berichtete Alvarotti von einem Gespräch der jungen Frau mit dem Konnetabel, welcher ihr von der Zufriedenheit des Königs über ihr tadelloses Verhalten, vor allem über ihr Desinteresse an queste barbuglierie della corte erzählt habe, und erläuternd merkt der Gesandte an:

[Queste barbuglierie] mò non sono altro che $i$ molti odij che sono tra damme, et damme et homini, et [homini] mescolandovi, et la reina, et madamma Margheritta, et uffitij che si fanno l'uno contra l'altro, chi per passione, et chi per malignità, et chi per causa de [chiavamenti], et detta Madama ch'è savia non porge ne occhio ne orecchie a nissuna di queste materie [et si governa saviamente con tutti] ${ }^{70}$.

69 Ercole d'Este an François de Lorraine, o.D. (1. H. Sept. 1548), Ferrara, in: BnF, Clair. 346, fol. 169r. Beim Herzog von Vendôme und der Prinzessin von Navarra handelt es sich um Antoine de Bourbon und Jeanne d'Albret. Die quattro righe der Prinzessin an ihren zuklinftigen Gemahl sind leider nicht erhalten.

${ }^{70}$ Giulio Alvarotti an Ercole d'Este, 7. Juli 1549, Paris, in: FONTANA, Renata di Francia, Bd. 2, S. 292. Die Wörter in Klammern wurden von mir anhand ASM, Canc. duc., Amb. Francia 46, lib. 27 ergänzt bzw. korrigiert. 
Agentin für den Herzog von Ferrara

und der Aufbau eines Beziehungsnetzes

Den Interessen des Vaters war die Prinzessin stets zu dienen bereit, und zeitweise war sie seine wichtigste Informationsquelle, etwa nachdem der Kardinal von Ferrara im Mai 1549 Frankreich verlassen hatte. Zwei Monate später erinnerte Ercoles Botschafter die Herzogin, daß sie nun, da sich Ippolito nicht mehr bei Hofe aufhalte, dem Vater ofter schreiben solle, wie sie es ihm bei ihrer Abfahrt aus der Heimatstadt versprochen habe. Tatsächlich unterrichteten Anna und ihr Gemahl den Gesandten uber die Vorgänge im Königreich, und $d a B$ es sich bei den Neuigkeiten nicht nur um Bagatellen handelte, geht aus ihrer Angst hervor, die Briefe könnten abgefangen werden ${ }^{71}$.

Am Initial der gedruckten Fassung von Annas Heiratsvertrag, das ein Bild Judiths zeigt, wie sie den Kopf des toten Holofernes in einen Sack packt, kann abgelesen werden, in welchem Maße Ercole von der Tochter in Frankreich politisches Engagement fur sein Herzogtum erwartete (Abb. 4). Die Figur der Judith war im 16. Jahrhundert vorwiegend positiv konnotiert, ihr Mord an Holofernes wurde als Sieg der Humilitas uber die Superbia gedeutet. Darstellungen der Judith-Geschichte finden sich oft im Zusammenhang mit furstlichen Heiraten, wie etwa die kostbaren Tapisserien zeigen, die zur Hochzeitsausstattung der Margarete von Österreich gehörten. Die Botschaft dieser Teppiche beinhaltete das Lob des Starkmutes, der Tatkraft und der Standhaftigkeit sowie die Aufforderung an die Braut, eine aktive politische Rolle zu übernehmen. Ähnliches läßt sich über eine für Johanna von Kastilien gefertigte Miniatur sagen, welche den Wunsch ausdrückte, die Prinzessin möge sich für ihr Volk mit dem gleichen Eifer einsetzen wie Judith es für das ihrige getan habe ${ }^{72}$. Die Person der Judith, verstanden als Idealtypus einer Herrscherin und Vorbild fur beherztes Handeln in schwierigen Zeiten, mußte fur eine junge Fürstin also eine nachahmenswerte Identifikationsfigur darstellen. Mit der Abbildung dieser alttestamentarischen Geschichte auf Annas Heiratsvertrag wollte der Herzog von Ferrara seine Älteste wohl zur Würdigung dieses vorbildlichen Verhaltens und mithin zu politischer Parteinahme in seinem Sinne animieren, nicht anders als August von Sachsen, der seiner Tochter

${ }^{71}$ Giulio Alvarotti an Ercole d'Este, 2. Juli 1549, Paris, in: ASM, Canc. duc., Amb. Francia 26; Anna d'Este an dens., 21. Apr. (1552), 1. Okt. o.J., in: Ibid. Cart. prin. est. $1568 \mathrm{~A} / 14$. Auch der Botschafter Venedigs schreibt des ofteren, er habe Neuigkeiten per bocca di Madama di Guisa, vgl. die Briefe von Marc' Antonio Barbaro in: SURIANO, BARBARO, Despatches.

${ }^{72}$ Birgit FRANKE, Barbara WELZEL, Judith. Modell für politische Machtteilhabe von Fürstinnen in den Niederlanden, in: Ulrike GAEBEL, Erika KARTSCHOKE (Hg.), Böse Frauen Gute Frauen. Darstellungskonventionen in Texten und Bildern des Mittelalters und der Frihen Neuzeit, Trier 2001, S. 133-153, hier S. 133-139. 
Anna zu ihrer Hochzeit im Jahre 1586 einen Kettenanhänger schenkte, der ebenfalls die Historia Judith darstellte. Tatsächlich sollten die Taten der Herzogin von Nemours in einem nach ihrem Tod entstandenen Lobgedicht mit denen einer Judith verglichen werden ${ }^{73}$.

Anfangs traf Anna die mit der Frankreichpolitik des Vaters in Zusammenhang stehenden Entscheidungen gemeinsam mit dem Kardinal und dem Botschafter von Ferrara. Im Sommer 1549, anläßlich der Schwierigkeiten um die Auszahlung der Mitgift, wurde Ercole hinsichtlich einer eventuellen ungewünschten Einmischung seiner Tochter in die Verhandlungen mit den Worten beruhigt, daß Anna sich ruhig in den Lauf der Dinge einschalten könne, einen Unterschied würde das nicht machen, da sie minderjährig sei ${ }^{74}$. Die Prinzessin forderte jedoch hartnäckig ihren Teil der Verantwortung, und besonders während ihrer Anfangszeit bei Hofe zeigte sie sich leicht gereizt, wenn man sie oder ihre Meinung zu ignorieren suchte: Io ho fatto l'ufficio con S.E. più destramente e leggiermente che ho potuto, ma non l'ho però potuto fare cosi leggiero ch'ella non si sia agravata, dicendo che non mira a queste cose, $e$ che facci pur con altri, schrieb etwa Alvarotti, e non con le $i^{75}$. Die Rolle der Vermittlerin, einer Art Botschafterin für die Interessen Ferraras, wurde der Prinzessin bald nicht mehr aberkannt. $\mathrm{Ob}$ sie den Vater über französische Sitten und Gebräuche informierte, bei Hofe das zweifelhafte Verhalten des Herzogs seiner Gattin gegenüber zu erklären versuchte oder Ercole vor Leuten warnte, die den Ruin seines Hauses zu sehen wünschten, stets war Anna darum bemüht, che tutto tornarà a benefitio di casa sua et alla fine a particolar contentezza della E..$^{76}$.

Mit nicht minder großem Eifer widmete sich die Prinzessin auch den französischen Angelegenheiten ihres Bruders Alfonso, kümmerte sich um dessen

${ }^{73}$ Zu Bedeutung und Rezeption der Judith-Figur: Elena CILETTI, Patriarchal Ideology in the Renaissance Iconography of Judith, in: Marilyn MIGIEL, Juliana SCHIESARI (Hg.), Refiguring Woman. Perspectives on Gender and the Italian Renaissance, Ithaca, London 1991, S. 35-70; Sara F. MATTHEWS GRIECO, Ange ou diablesse. La représentation de la femme au $\mathrm{XVI}^{\mathrm{e}}$ siècle, Paris 1991, S. 153-160; Jutta SEIBERT, Art. "Judith«, in: Engelbert KIRSCHBAUM (Hg.), Lexikon der christlichen Ikonographie, Bd. 2, Rom u.a. 1970, Sp. 454458, hier Sp. 455. Zu Anna von Sachsen: ROGGE, Töchter, S. 250f. Das Lobgedicht: Severin BERTRAND, Oraison funebre sur le trespas de tres-haulte, tres-illustre et tres-vertueuse Princesse Anne d'Est', Duchesse de Chartres, de Guyse, Nemours, Genevois, etc., Paris 1607, fol. 16v.

${ }^{74}$ Caso in italiano; Niccolò Bendidio an Ercole d'Este, 28. Apr. 1549, Paris, in: ASM, Canc. duc., Amb. Francia 52.

${ }^{75}$ Giulio Alvarotti an Ercole d'Este, 5. Juli 1549, Paris, in: ASM, Canc. duc., Amb. Francia 26.

${ }^{76}$ Anna d'Este an Ercole d'Este, 18. Mai (1550), 5. Mai 1556, 10. Juli 1556, in: ASM, Canc. duc., Cart. prin. est. 1568 A/14; Ercole d'Este an Giulio Alvarotti, 3. März 1555, in: FonTANA, Renata di Francia, Bd. 3, S. XVII. 
Geldgeschäfte und Liebeshändel ${ }^{77}$. Selbst nach dem Tod des Vaters verringerte sich ihre Loyalität dem neuen Herzog und der Heimatstadt gegenüber nie, und nicht einmal die größten Spannungen in der Beziehung der Geschwister konnten etwas daran ändern, daß Anna sich immer auch als Este fuhlte. Casa Guisa e Madama di Nemours fanno quanto possono per Ferrara, berichteten Beobachter nicht selten über ihre Bemühungen zugunsten des Bruders ${ }^{78}$. Vor allem in den $1570 \mathrm{er}$ Jahren, als die Erhebung des Herzogs von Florenz zum Großherzog durch den Papst von Alfonso nicht, vom französischen Hof aber doch anerkannt wurde und sich in der Folge erhebliche Vortrittsstreitigkeiten zwischen den Gesandten der beiden Fürsten ergaben, trat Anna stets für den Bruder ein. So redeten sie und ihr ältester Sohn Anfang des Jahres 1580 so lange auf den König ein, bis der ferraresische Botschafter auf den ihm ihrer Meinung nach zustehenden Platz bei den Neujahrsfeierlichkeiten gesetzt wur$\mathrm{de}^{79}$. Obwohl die der Prinzessin vorgeworfenen, gegen den Großherzog gerichteten Intrigen nicht selten den Zorn der Königinmutter herausforderten, bemühte sich der französische Hof doch, Alfonso das Leben nicht allzu schwer zu machen, und dies, wie in Venedig zu erfahren war, aus Respekt vor dem Kardinal von Este und der Herzogin von Nemours, che piglia sempre la sua protezione. Noch Jahre später unterrichtete Anna den Bruder darüber, wie hoch er in der Gunst des Königs stünde und bot ihm ihre Kinder, Enkelkinder und tous nos amis an, pour lui faire le trèshu[m]ble et fidelle service ${ }^{80}$.

Doch nicht nur den Mitgliedern der Familie der Este galten die Bemühungen der Herzogin, ihren Einfluß verwandte sie auch für alle anderen Personen, die sich in ihren Schutz begaben. Li faccio con queste mie [lettere] riverenza, $e$ tornandoli a memoria la servitù mia la prego a tener la protection mia, sì come per la sua benigna merce è stata sempre solita, heißt es in einem der vielen an sie gerichteten Bittbriefe ${ }^{81}$. Ihr ehemaliger Kurator und späterer königlicher Kanzler Michel de L'Hospital wandte sich an die Prinzessin mit der Bitte, in den Kreis ihrer Klienten aufgenommen zu werden: Einst sei er ihr Tutor und sie sein Mündel gewesen, schreibt L'Hospital, nun sollten sie die Rollen tauschen. Die Vertrautheit, welche zwischen dem jungen Mädchen und

\footnotetext{
${ }^{77}$ Anna d'Este an Alfonso d'Este, 1. Sept. o.J. (1556?), 7. Okt. 1559, in: ASM, Canc. duc., Cart. prin. est. $1568 \mathrm{~A} / 14$.

${ }^{78}$ Gianmaria Petrucci an Francesco de' Medici, 24. Mai 1571, in: DESJARdiNS (Hg.), Négotiations diplomatiques, Bd. 3, S. 670f.

${ }^{79}$ Sinolfo Saracini an Francesco de' Medici, 3. Jan. 1580, in: DESJARDINS (Hg.), Négotations diplomatiques, Bd. 4, S. $277 \mathrm{f}$.

${ }^{80}$ Sigismondo Cavalli, Relazione für die Serenissima, 1574, in: Luigi FIRPO (Hg.), Relazioni di ambasciatori veneti al Senato, Bd. 5: Francia (1492-1600), Turin 1978, S. 705; Anna d'Este an Alfonso d'Este, 10. Sept. 1596, Saint-Maur-des-Fossés, in: ASM, Canc. duc., Cart. prin. est. 1458/22.

${ }^{81}$ Cornelio Bentivoglio an Anna d'Este, 18. Nov. 1553, Siena, in: BnF, Clair. 347, fol. $115 r$.
} 
ihrem Kurator während der gemeinsamen Reise nach Frankreich entstanden war, sollte für seine Karriere eine wichtige Rolle spielen. In einer Jahre später an die Herzogin von Nemours gerichteten Epistel erinnerte L'Hospital daran, $\mathrm{da} ß$ er von den Diensten für die ferraresische Prinzessin direkt in die des Konigs und der Königin gewechselt war, und tatsächlich scheint Anna neben Katharina von Medici und Margarete von Valois die fur seine Karriere wichtigste Dame gewesen zu sein $^{82}$.

Auch Annas Gefahrtin aus Jugendzeiten, Olympia Morata, die mit ihrem Gatten mittlerweile in Heidelberg lebte und von dort aus das Schicksal der französischen Protestanten verfolgte, wandte sich an die Herzogin mit einer Bitte, der nachzukommen ein nicht geringes Risiko für die Gemahlin des wichtigsten Katholikenfuhrers darstellen mußte. Den bedrängten Hugenotten solle sie ihre wahre Überzeugung offenbaren, sie vor dem König rechtfertigen und um Gnade für sie bitten, denn durch ihr Schweigen würde die Prinzessin den Morden und der Verfolgung zustimmen, gar den Anschein erwecken, mit auf die Vernichtung ihrer Glaubensgenossen zu sinnen. Eventuelle Einwände ihrer fruheren Gefährtin, sich den Zorn des Königs, ihres Gatten und vieler anderer zuzuziehen, entkräftete Olympia mit dem Hinweis: Cogita satius esse, te odio esse hominibus, quam Deo, qui non solum corpus sed etiam animam, perpetuo igni, cruciare potest ${ }^{83}$.

Ihre Wünsche und die der anderen präsentierte die Herzogin dem König in der Regel nicht persőnlich, denn bald schon hatte sie die Mechanismen des Hofes verstanden und ihnen zu folgen gelernt. Der Schlüssel zur Erlangung des Gewunschten lag auch furr die italienische Prinzessin bei Diane de Poitiers oder, nach Heinrichs Tod, bei der Königinmutter. Ercoles Agenten begaben sich daher normalerweise zunächst zu Anna, die dann mit Gemahl und Schwager sprach, welche die Angelegenheit wiederum der Herzogin von Valentinois vortrugen; oder aber sie sprach, vor allem in späteren Jahren, selbst mit Katharina, damit diese auf ihren Sohn einwirke ${ }^{84}$. Tatsächlich verstand die Prinzessin es, sich mit den jeweils herrschenden Damen zu verständigen. So berichtet Alvarotti, wie er einen ganzen Nachmittag auf die Hochschwangere gewartet habe, die sich in einer Unterredung mit Diane de Poitiers befand. Am selben Abend sollte Anna ihr erstes Kind zur Welt bringen, was sie jedoch nicht von stundenlangen Verhandlungen abgehalten zu haben scheint: quan-

${ }^{82}$ DUPRÉ LASALE, L'Hospital, S. 133f.; KIM, L'Hôpital, S. 26. Die Epistel von 1572 in: Michel de L'Hospital, Euvres completes, Bd. 3: Poematum: Epistolarum, hg. v. P. J. S. DUFÉY, Paris 1825, ND 1968, S. 491-494, hier S. 492.

${ }^{83}$ Olympia Morata an Anna d'Este, o.D. (Winter 1554 aus Heidelberg), in: MORATA, Opere, S. 116.

Giulio Alvarotti an Ercole d'Este, 29.-30. Jan. 1549, Poissy, in: FonTANA, Renata di Francia, Bd. 2, S. 284 u. ASM, Canc. duc., Amb. Francia 45; Anna d'Este an Alfonso d'Este, 7. Okt. 1559, Blois, in: Ibid. Cart. prin. est. 1568 A/14. 
tunque io la attendessi tanto che si facea scuro quando partei dal suo loggiamento, ove ella non era ancho retirata di su la predetta de Valentinois ${ }^{85}$.

Rund zwanzig Jahre später war die Prinzessin wieder schwanger und wieder bei nächtelangen Unterredungen mit der Königinmutter anzutreffen. Ihre Nähe zu Katharina von Medici, ebenso wie zu der jeweiligen Königin, war ein wichtiger Faktor für Annas Rolle als Sammelpunkt von Informationen und Gerüchten. Wendungen wie wio so che Madama di Guisa ha detto haver dalla Regina che..." finden sich in den Briefen vieler Berichterstatter ${ }^{86}$. Auch die Schwäger Charles und Louis spielten für ihre politische Stellung eine entscheidende Rolle, wobei »Politik« hier als Einflußnahme mit dem Ziel verstanden werden muß, die Möglichkeiten der eigenen Familie zur Machtausübung sowie zur Teilnahme an der Gestaltung des Hofes und den Entscheidungen des Königs zu vergrößern. Die beiden Kirchenfürsten sorgten dafür, daß sich die Herzogin zu den am besten informierten Damen Frankreichs zählen konnte, unterrichteten sie über die Sitzungen des königlichen Rates, uber die Pläne des Königs sowie über Neuigkeiten von der Hofgesellschaf ${ }^{87}$.

\section{Möglichkeiten und Grenzen politischen Handelns der Herzogin}

Die Herzogin von Guise interessierte sich nicht nur fur das Wohl von Familienangehörigen oder Klienten, auch an den großen politischen Diskussionen des Hofes nahm sie teil. Nach dem Tode Pauls III. unterhielt sie sich in aller Vertraulichkeit mit Heinrich über das Konklave und die zu unterstützenden Kardinäle, und während der letzten Phase des Konzils von Trient war die Prinzessin gemeinsam mit dem König und anderen Großen des Reiches bei einer Diskussion mit dem englischen Botschafter über Sinn und Unsinn der Entsendung von Klerikern anwesend ${ }^{88}$. So hatte sich die Herzogin bald eine Reputation erworben, die ihr Verständigkeit und Fleiß bescheinigte: Con la Regina Madre bisogna negoziare e ambular via; e con madama de Guise si può andare per trattenimento, hieß es im Dezember 1559 über Annas Făhigkeit zu verhandeln, und einige Zeit spăter: Madama de Nemours è qui; lavora

\footnotetext{
${ }^{85}$ Giulio Alvarotti an Ercole d'Este, 31. Dez. 1549, Melun, in: ASM, Canc. duc., Amb. Francia 26, fol. 152v.

${ }^{86}$ Gianmaria Petrucci an Francesco de' Medici, 23. Juli 1572, in: DESJARdINS (Hg.), Négotiations diplomatiques, Bd. 3, S. 799; Marc' Antonio Barbaro an die Serenissima, 9. Nov. 1562, Paris, in: SURIANO, BARBARO, Despatches, S. LXXIV.

${ }^{87} \mathrm{Vgl}$. die Briefe von Charles und Louis de Lorraine an Anna d'Este, in: LORRAINE, Lettres u. BnF, Fr. 3231-3232.

${ }^{88}$ Giulio Alvarotti an Ercole d'Este, 30. Nov. 1549, Paris, in: ASM, Canc. duc., Amb. Francia 26; Nicolas Throckmorton an Elisabeth I., 17. Apr. 1562, Paris, in: Calendar Elizabeth, 1866, S. 603.
} 
da valente, e ci fa testa. In fatti vuò dire il vero; ci sono alcune donne che valgono per molti uominis.

1559 war Anna bei den Verhandlungen anwesend, die zum Frieden von Cateau-Cambrésis fuhren sollten. Gemeinsam mit Katharinas Tochter Claude reiste sie Ende Februar in die Niederlande, wo sie von Christine von Dänemark empfangen und reich beschenkt wurde. Die Anwesenheit der Damen scheint den Zweck verfolgt zu haben, die Verhandlungen zu einem raschen Abschluß zu bringen: Vedendosi queste amorevolezze, si spera assai che la pace habbia ad ogni modo da essere, wurde etwa nach Mantua berichtet, $e$ par' non si attendi ad altro che a far' allegrezze e banchetti tra quelli signori che sono al parlamento ${ }^{90}$. Viele Jahre schon kannten sich Anna und Christine, die beide in das Haus Lothringen eingeheiratet hatten. Brantôme berichtet von ihrem ersten Treffen, bei dem sich die Damen so aufmerksam gemustert hätten, que l'une et l'autre ne se pouvoient assez regarder, tant elles furent fixes et attantives à s'entrevoir ${ }^{91}$. Die bei den Friedensverhandlungen anwesenden Spanier waren begeistert von der Herzogin von Guise. Philipp II. ließ ihr kostbaren Schmuck uberreichen, und die Prinzessin kehrte reich beschenkt an den Hof zurück:

Hoggi deve arrivare in corte di ritorno Madama la duchessa di Lorena [Claude de Valois] e Madama di Guisa, quale ha hauto dalla Maestà del re Filippo un presente de gioie de 15000 scudi, e da Madama di Lorena [Christine von Dänemark] il carcame che già la Maestà dell' Imperatore gli donò quando fu duchessa di Milano. Ha donato anchor a Madama di Guisa goie e perle per 4000 scudi ${ }^{62}$.

Zum französischen Botschafter in Spanien stand Anna in jenen Jahren in regelmäßigem Kontakt, wie eines der Schreiben zeigt, in dem sich der Gesandte bei der Herzogin für das Ausbleiben seiner Briefe entschuldigt. Und bevor sich der französische Hof 1565 in Bayonne mit Katharinas Tochter Elisabeth traf, erkundigte sich der Gesandte bei Anna, welche Mitbringsel sie von der iberischen Halbinsel wünsche. Auch bei diesem Treffen hinterließ die Herzogin von Guise einen bleibenden Eindruck bei den anwesenden Spaniern, die große Bewunderung für sie bezeugten, autant pour sa beauté et belle grace

${ }^{89}$ Alfonso Tornabuoni an Cosimo de' Medici, 12. Dez. 1559, in: DESJARDINS (Hg.), Négotiations diplomatiques, Bd. 3, S. 407; Filippo Cavriani an Bartolomeo Concini, 19. Juli 1572, in: Ibid. S. 798.

${ }^{90}$ Ercole Strozzi an Guglielmo Gonzaga, 22. Febr. 1559, La Ferté-Milon, in: ASMan, AG 651; Francesco Beccio an Bernardino Belliano, 10. März 1559, Paris, in: Ibid. Zu Annas Anwesenheit in Cateau-Cambrésis: ROMIER, Origines, Bd. 2, S. 341.

${ }^{91}$ BRANTÓME, Recueil des Dames, S. 520.

${ }^{92}$ Ercole Strozzi an Guglielmo Gonzaga, 16. März 1559, La Ferté-Milon, in: ASMan, AG 651 . 
que pour porter titre de femme de $M$. de Guyze; et ne l'appelloient que sla muger d'aquel gran ducque de Guysar'.

Kaum ein politisches oder gesellschaftliches Ereignis der folgenden Jahre, in dessen Erzählung der Name der Prinzessin nicht gefallen wäre. Zwar hing ein Teil ihres Einflusses und ihrer Handlungsmöglichkeiten vom Geschick ihres Gemahls ab, wie etwa Ende der 1550er Jahre, als die Guise bei Hofe hoch im Kurs standen und daher auch Anna größere Macht besaß: Die Herzogin, hieß es tuber sie, adesso puo assai, doch war sie nicht zwangsweise an die Familie ihres Mannes gebunden. Während die Guise als naturalisierte Ausländer auf das Wohlwollen des Königs angewiesen waren, blieb die Prinzessin, als Tochter einer Königstochter selbst von königlichem Blut, von den Schwankungen der Gunst des jeweiligen Herrschers eher unberürt, und ihre Stellung bei Hofe hing nach dem Tode Heinrichs mehr von ihrem Verhältnis zur Königinmutter ab. Auch mußte sie mit der Politik ihres Gemahls und dessen Brüdern nicht unbedingt in allen Punkten ubereinstimmen, ihre grundsätzliche Loyalität den Guise gegenüber wurde aber nie ernsthaft in Frage gestellt ${ }^{94}$.

So setzte Anna sich nach der sogenannten Verschwörung von Amboise gemeinsam mit Maria Stuart und einigen anderen Damen des Hofes für die Freilassung der Gefangenen ein, während ihr vom König mit dem Amt des lieutenant général und außergewöhnlichen Kompetenzen versehene Gemahl die Todesurteile vollstrecken ließ. Die dem Sekretär von François de Montmorency, Louis Régnier de La Planche, zugeschriebene Geschichte Frankreichs berichtet, der Herzog von Guise habe seine Frau gezwungen, bei den Exekutionen zuzusehen. Jacques-Auguste de Thou, gemäßigter Katholik und Gegner der Guise und deren Ligue, widerspricht in seinem rund vierzig Jahre nach den Vorfällen in Amboise verfaßten Geschichtswerk dieser Darstellung:

Tous les Seigneurs et Dames de la Cour étoient aussi aux fenêtres pour voir les exécutions. La seule Anne d'Est Duchesse de Guise, Dame d'un esprit doux et humain, n'y parut pas. Elle avoit été élelvée à Ferrare par Renée de France, sa mere, dans cette même doctrine $q u$ 'on persécutoit alors, et avoit eu pour confidente Olimpia-Fulvia Morata, fille célèbre en Italie par son grand sçavoir, et imbûë des nouvelles opinions. On assure qu'en cette occasion la Duchesse de Guise versa bien des larmes, et dit à la Reine-mere que si elle vouloit conserver le Roi et l'Etat, elle devoit empêcher le supplice des innocens'.

${ }^{93}$ BRANTÔME, Euvres complètes, S. 102f. Die Briefe des Gesandten: Jean Ébrard an Anna d'Este, 7. Okt. 1564, 21. Jan. 1565, in: Jean Ébrard, seigneur de SAINT-SULPICE, Ambassade en Espagne de 1562 à 1565, et mission de ce diplomate dans le même pays en 1566, hg. v. Edmond CABIÉ, Paris 1903, S. 306, 336f.

94 Giovambattista Gondi an Renée de France, 24. Okt. 1557, Paris, in: BnF, Fr. 3043, fol. 99r.

${ }^{95}$ THOU, Histoire universelle, 1742, Bd. 2, S. 773; (Louis Régnier de LA PLANCHE), Histoire de l'Estat de France, tant de la Republique que de la Religion: Sous le Regne de François II., 
Im Laufe der Zeit ist das Engagement der Damen vergessen worden, so daß in der bis heute immer noch umfassendsten Darstellung der Ereignisse um die Verschwörung von Amboise zu lesen ist: „Personne ne prit la défense des condamnés ${ }^{96}$. Die Zeitgenossen ignorierten die Herzogin von Guise jedoch keineswegs. Während sie als Tochter der Hugenottenbeschützerin Renée de France in den Berichten mancher gemäßigter oder reformatorisch Gesinnter geschont wurde, sahen andere in ihr schlichtweg eine Mitwirkende an der tyrannie de ceux de Guise und bezogen sie in ihre Polemiken ein ${ }^{97}$. So François Hotman, der in seiner 1560 anonym veröffentlichten »Epistre envoyée au Tigre de la France« dem Kardinal von Lothringen sexuellen Verkehr mit seiner Schwägerin vorwarf, eine Anschuldigung, die von d'Aubigné in seinen "Misères« wieder aufgegriffen und von den beiden 'Angeklagten< laut Brantôme mit derartiger Bestürzung zur Kenntnis genommen wurde, qu'ils en cuiderent desesperer ${ }^{98}$.

Geschont wurde Renées Tochter in den aus hugenottenfreundlichen Federn stammenden Berichten uber den als Massaker von Vassy bekannten Zusammenstoß Anfang März 1562 von Besuchern eines reformierten Gottesdienstes mit den Mănnern des Herzogs von Guise. Während in einer dieser Schriften, die wahrscheinlich aus der Feder des ehemaligen Predigers der Gemeinde Vassy stammt, die Schuld an der Eskalation der Gewalt eindeutig François zugewiesen wird, erscheint dessen Gemahlin als Retterin der bei dem Gemetzel anwesenden schwangeren Frauen:

Le Duc estoit luy-mesme en la grange, avecques son espée [...] en la main, commandant à ses gens de tuer, et nommément les jeunes gens. Et, sur la fin, dit qu'on laissast les femmes grosses; criant après ceux qui estoyent sur les eschaffanx, qui esforçoient de se sauver par ledit toict: En bas canailles, en bas; et usant de grandes menaces. Ce qui le meut lors d'ordnonner qu'on laissast les femmes grosses, fut par le moyen de la Duchesse sa femme, laquelle, passant auprès des murailles dudit Vassy et oyant un si grand bruit et clameur de ces pauvres gens et le son des hacquebutes et pistolets, envoye en diligence vers le Duc son mary, le supplier de cesser sa persécution, de peur des femmes grosses 99 .

o.O. 1576, S. 224f. Der Bericht über Annas Engagement für die Freilassung der Gefangenen: VIEILLEVILLE, Mémoires, Bd. 2, S. 424f.

${ }^{96}$ Lucien ROMIER, La conjuration d'Amboise. L'aurore sanglante de la liberté de conscience, le règne et la mort de François II, Paris 1923, S. 117.

${ }^{97}$ So im Titel einer von den »Verschwörern« an den König gerichteten Schrift, in: JOUANNA u.a., Histoire et Dictionnaire, S. 66.

98 (François HOTMAN), Le Tigre de 1560, hg. v. Charles READ, Paris 1875, ND 1970, S. 43f.; Agrippa d'AUBIGNE, Les Tragiques, hg. v. Frank LESTRINGANT, Paris 1995, S. 105; BRANTÓME, Recueil des Dames, S. 636.

${ }_{99}$ Discours entier de la persecution et cruauté exercée en la ville de Vassy par le Duc de Guyse, le 1. de Mars 1562, o.O. o.J. [1562], in: Mémoires de Condé, Servant d'Éclaircissement et de Preuves à l'Histoire de $\mathrm{M}$. de Thou, Contenant ce qui s'est passé de plus mémorable en Europe, Bd. 3, London 1743, S. 124-149, hier S. 137. Zu Autorschaft und Datierung 
Auch der zeitgenőssische protestantische Geschichtsschreiber Jean de Serres läßt den Herzog von Guise, von seiner Gattin um Gnade fur die Unglücklichen gebeten, das Morden sofort beenden, und selbst Théodor de Bèze, der ansonsten nicht immer gut auf Renées Tochter zu sprechen ist, folgt in seiner "Histoire Écclesiastique» dieser Darstellung ${ }^{100}$. Jacques-Auguste de Thou erklärt Annas Bemühen zur Rettung der Frauen von Vassy wie auch in dem bereits zitierten Kapitel über die Hinrichtungen in Amboise mit dem Hinweis auf den "gutigen « und "sanften" Charakter der Prinzessin und ihre Erziehung durch eine der Reformation zugeneigte Mutter. Dabei vergißt er nicht zu erwăhnen, daß zum Zeitpunkt des Geschehens auch die Herzogin selbst als den Protestanten freundlich gesonnen galt:

Le bruit fut si grand, qu'il vint jusqu'anx oreilles d'Anne d'Este, Duchesse de Guise, qui étoit portée en litiere [...]. Cette Dame se douta de ce qui étoit arrivé. Pleine de bonté et de douceur, elle passoit d'ailleurs pour n'être pas ennemie des Protestans; au moins Renée Duchesse de Ferrare sa mere ne lui avoit pas appris à les haïr. Elle envoya donc aussi-tôt un exprès au Duc son mari, pour le prier d'épargner le sang de ces malheureux ${ }^{101}$.

Die anhand von Quellen nicht zu belegende Behauptung, Katharina von Medici habe ihre Freundin im Februar 1563 nach Orléans geschickt, um sie als Fürsprecherin beim Herzog von Guise einzusetzen und die Stadtbewohner vor den Plünderem aus dem katholischen Lager zu bewahren, zeigt schließlich, daß sich Annas Ruf als Beschützerin unschuldiger Hugenotten bis ins 19. Jahrhundert hinein gehalten hat ${ }^{102}$.

Möglichkeiten und Grenzen politischen Handelns der Prinzessin können schließlich am ,Fall Boturnio Boturnei erklärt werden. Der Theologe hatte spätestens seit Mitte der 1530er Jahre Renées Hof angehört und war auf deren Wunsch der ältesten Tochter nach Frankreich gefolgt. Bei dem unter Annas Reisebegleitern verzeichneten omonier dürfte es sich also um ihn handeln. Im

des Textes: Philip BENEDICT u.a., Graphic History: What Readers Knew and Were Taught in the Quarante Tableaux of Perrissin and Tortorel, in: French Historical Studies 28 (2005) S. 175-229, hier S. 190. Das Gemetzel fand in einem als "Scheune« bezeichneten Gebalude statt. Die Herzogin war zu diesem Zeitpunkt selbst seit einigen Wochen schwanger.

100 Jean de Serres, De statu religionis, in: Alphonse de RUBLE, L'assassinat de François de Lorraine, duc de Guise, 18 février 1563, Paris 1897, S. 171-173; (Théodor de BĖZE), Histoire Écclesiastique des églises reformées au royaume de France, Bd. 1, Antwerpen 1580, S. 727. François de Lorraine selbst versuchte, mit Hinweis auf die Anwesenheit seiner Gattin und eines ihrer Kinder seine Unschuld zu beweisen: François de Lorraine an Christoph von Wurttemberg, 17. Mărz (1562), Paris, in: François de LORRAINE, duc de Guise, Correspondance avec Christophe, duc de Wurtemberg. Deuxième série: Massacre de Vassy (Mars-mai 1562), in: Bulletin de la Société de l'histoire du protestantisme français 24 (1875) S. 209221, 499-513, hier S. 216.

${ }^{101}$ THOU, Histoire universelle, 1742, Bd. 3, S. 130.

${ }^{102}$ RUBLE, Assassinat, S. 40f. 
Juli 1552 verschaffte ihm die Herzogin von Aumale den Posten des Priors des Hôtel-Dieu in Provins. Bald schon wurden Boturnio Amtsmißbrauch, unzüchtige Lebensweise und Häresie vorgeworfen, und gemeinsam mit dem Rat der Stadt versuchte der lokale Klerus, ihn von seinem Posten zu entfernen. Inwieweit die erhobenen Anschuldigungen gerechtfertigt waren, ist kaum festzustellen, da Claude Hatons nicht unvoreingenommene Memoiren die einzig verfuigbare Quelle darstellen. In einer mit »Eretici che erano in Ferrara al tempo di Madama Renea « uberschriebenen Namensliste heißt es jedenfalls uber Boturnio: Si può dire che lui parimente non camini bene nella religione ${ }^{103}$. Mit Annas Hilfe gelang es ihm jedoch, sich einer drohenden Gerichtsverhandlung zu entziehen. Die Herzogin, die in dieser Zeit ihre ersten Erfahrungen mit dem französischen Gerichtswesen zu sammeln begann, hatte ihrem Schutzbefohlenen den Titel eines königlichen Almosenpflegers verschaff, wodurch ihn betreffende Angelegenheiten direkt dem geheimen Rat des Herrschers unterstellt wurden. Bis hierhin reichten also die Möglichkeiten und Fähigkeiten der Prinzessin: Sie hatte die Macht, ihren Klienten trotz eventueller Widerstände die gewünschten Posten zu verschaffen, sie konnte sich gegen die Entscheidungen einer Stadt durchsetzen, und sie scheint strategisches Talent sowie das Wohlwollen des Königs besessen zu haben. Als der Herzog von Guise jedoch drei Jahre spăter wăhrend seines Italienfeldzuges mit dem Papst zusammentraf und dieser ihm Vorhaltungen ob der Situation in Provins machte, konnte die Prinzessin ihren Schützling nicht mehr retten. Von ihrem Gemahl erhielt sie den Befehl, den Italiener aus ihren Diensten zu entlassen, sus peine de s'en repentir elle-mesme mès qu'il [François] feust de retour en France, et luy [Boturnio] avec si encores y estoit trouvé. Eine eindeutige Anweisung ihres Gatten, erteilt aufgrund einer päpstlichen Beschwerde - das überstieg Annas Kompetenzen. Erst Jahre später, als Witwe, würde die Herzogin die ihr zur Verfügung stehenden Möglichkeiten voll ausschöpfen können ${ }^{104}$.

\section{Taktik und Rhetorik: Ein Brief an den Bruder}

Die rhetorischen Fähigkeiten, die Anna im Laufe der Jahre entwickelte, die Art und Weise ihrer Argumentation sowie ihre Strategien zur Erreichung des Gewünschten, können aus einem ihrer an den Bruder Alfonso gerichteten Schreiben abgelesen werden. Verfaßt wurde dieser Brief in einer Zeit, als das Verhältnis zwischen Ercole und dem französischen Hof gestört war, da in

${ }^{103}$ Die 1554 erstellte Liste in: FonTANA, Renata di Francia, Bd. 3, S. XXXII. Meine Suche nach dem Original dieses Dokuments im Archiv von Modena blieb ergebnislos.

${ }^{104}$ HATON, Mémoires, Bd. 1, S. 9-12, 76f., Zitat S. 76. Vgl. John A. TEDESCHI, Art. "Boturnei, Boturnio dei«, in: Dizionario biografico degli Italiani, Bd. 13, Rom 1971, S. 509. 
Frankreich das Scheitern des Italienfeldzuges des Herzogs von Guise im Jahre 1557 dem Unwillen Ferraras zur Kooperation zugeschrieben wurde. Als sich Ercole und Philipp II. dann im Mărz des folgenden Jahres auf einen Friedensvertrag einigten, durch den das Herzogtum Ferrara unter spanischen Einfluß zu geraten drohte, und zur gleichen Zeit die bevorstehende Heirat des Erbprinzen Alfonso mit einer Tochter des Herzogs von Florenz in Frankreich bekannt wurde, hatte Anna ihre völlige Kompromittierung bei Hofe zu befuirchten. Aus dieser Situation heraus entstand der Brief an den Bruder, in dem die Prinzessin eindringlich vor den Folgen einer in ihren Augen unwürdigen Verbindung warnte, die daruber hinaus das gute Verhältnis Ferraras zu Heinrich II. zerstören würde. Anna wußte, welche Bedeutung der König und sein Hof für den Prinzen hatten, der schon einige Jahre zuvor nach Frankreich sgeflohen um sich Ruhm und Ehre zu erwerben und den drastischen Sparmaßnahmen des Vaters zu entgehen, die sich auch auf das Budget des Sohnes auswirkten (Abb. 5) ${ }^{105}$.

In zwei Richtungen zielt die Argumentation des Briefes: Die Herzogin beschwört das Ende des freundschaftlichen Verhältnisses zwischen dem Bruder und dem französischen Hof, sollte die unerwünschte Heirat zustande kommen, und sie diskreditiert die Familie der Braut, die einer Verbindung mit den Este nicht würdig sei. Daruber hinaus ist der Ton des Briefes sehr emotional gehalten; Anna verweist wiederholt auf ihre eigene zuklinftige, angesichts der bevorstehenden Hochzeit traurige Situation. Stilistisch unterscheidet sich dieses Schreiben deutlich von den üblichen Briefen sowohl der Herzogin als auch anderer Fürstinnen und Fürsten der Zeit. Formelhafte Wendungen und Wortpaarungen fehlen fast völlig, so daß die sonst erreichte Verlangsamung der Lesegeschwindigkeit hier nicht stattfindet. Wie in einem starken Gefühl der Verärgerung zu Papier gebracht erscheinen die Zeilen, die mit den in anderen Briefen $\mathrm{zu}$ findenden starren Formulierungen nicht $\mathrm{zu}$ vergleichen sind, sondern einen stärker appelativen und emotionalen Charakter besitzen ${ }^{106}$.

Tout le monde a trouvé le plus estrange qu'il est possible le mariage que l'on dit que l'on traytte de vous et d'une des filles du duc de Floranse, que y'ai trouvé, quant à moy, si estrange que ye ne le croyre yames, beginnt die Prinzessin, nachdem sie, im eigenen sowie im Namen des Königs, den kürz-

${ }^{105}$ Der Brief: Anna d'Este an Alfonso d'Este, 21. Apr. 1558, in: ASM, Canc. duc., Cart. prin. est. $1568 \mathrm{~A} / 14$, hier alle folgenden Zitate. Zu den Spannungen zwischen den Höfen von Frankreich und Ferrara und zu Annas Situation in dieser Zeit: Giovanni Soranzo, Relazione furr die Serenissima, 1558, in: FIRPO (Hg.), Relazioni di ambasciatori, S. 410-412; ROMIER, Origines, Bd. 2, S. 108-224; CHIAPPINI, Estensi, S. 315 f.

${ }^{106}$ Eine Analyse von französischen Briefen des 16. Jh.: NEUSCHEL, Honor, S. 103-107. Vgl. Gerhard FOUQUET, Fürsten unter sich - Privatheit und Offentlichkeit, Emotionalität und Zeremoniell im Medium des Briefes, in: Cordula NolTE u.a. (Hg.), Principes. Dynastien und Hoffe im späten Mittelalter, Stuttgart 2002, S. 171-198, hier S. 191. 
lich abgeschlossenen spanisch-ferraresischen Friedensvertrag gelobt hat, was wohl beweisen soll, daß sie und der Hof, seit der gescheiterten Italienexpedition ihres Gatten, nicht alle den Herzog von Ferrara und seinen Staat betreffende Neuigkeiten ablehnten, sondern sehr wohl zwischen Vor- und Nachteilen zu unterscheiden wüßten. Der Hinweis, daß sowohl sie selbst als auch der gesamte Hof von der Neuigkeit uber das Heiratsprojekt irritiert sei, entspricht der Taktik Annas, die Ablehnung als allgemeinen Konsens darzustellen, l'oppinion de tout le monde, und den eigenen Standpunkt hervorzuheben, dabei aber den eventuellen Vorwurf, nur den persönlichen Interessen zu dienen, strikt zuruckzuweisen: Si Monsieur mon mari et moy ne savyons le mal qui vous en peut avenir, nous ne le vous mandrions point, car pour notre particulier sella ne nous inporte point. In den schwärzesten Farben schildert Anna dem Bruder seine Zukunft am französischen Hof und die seines Gefolges: yames prinse ne fust pis venu ne vos gens plus mal recceu que vous serres, si sella avient. Der Grund dieser allgemeinen Ablehnung läge, so die Herzogin, in dieser Verbindung tant contre votre honneur et contre la vollonté du roy, zurückzufuhren auf die Unterlegenheit der Familie der Braut im Vergleich zu der des Bräutigams, die der Tochter eines Königs wlirdig sei:

Il y a si grand differense de leur mayson et della nôtre et de l'alliance que yusque à cest heure nous avons eu, qui ont esté de tant de fille de roys, que quant à moy, ancorre que ye soys de moyndre de notre mayson, mon ceur ne sarroyt porter d'avoyr pour ma seur aysnée une telle fille et pour mays [mes] alliés des marchans qui ne sont point di[g]nes de se nommer nos serviteur[s]. Et ancorre san[s] mille autre[s] inperfections que l'on m'a asseuré que toutte leur rasse ha, et le dit-on tout communement parmi tous les grans de sete conpagnie, qui est qu'elle[s] sont non seullement bossue[s], mays si fort bides qu'elles en sont monstrueuse[s] et font peur aus gens.

Ihren eigenen Standpunkt mit drastischen Worten zu beschreiben vergißt Anna nicht. Dabei unterlaßt sie es auch nicht, ihren Bruder über die wahren, hinter höfischer Dissimulation versteckten Gedanken Heinrichs aufzuklären. Zwei Jahre später sollte sie sich einer ähnlichen Strategie bedienen, als sie Alfonso, der mittlerweile selbst Herzog von Ferrara war, in gran confidenza wissen ließ, die französischen Herrscher zeigten sich zwar nell' istrinseco mit ihm zufrieden, seien es aber in effetto keineswegs ${ }^{107}$. Auf diese Weise konnte Anna das Vertrauen, welches der König ihr entgegenbrachte, demonstrieren und gleichzeitig ihren Worten größere Glaubhaftigkeit verleihen:

Ye voys qui viendra entre le roy et vous de si grand deffianse, que ye croy que pour le plus grand heur que ye me saroys souhayter, s'il est a[i]nsi, ses [c'est] de mourir, pour ne voyr point tant de maus que seus que ye voys presanter pour vous et toutte ma mayson, si sella

${ }^{107}$ Giulio Alvarotti an Alfonso d'Este, 2. Mai 1560, Amboise, in: ASM, Canc. duc., Amb. Francia 36, fasc. II, fol. 24r. 
avyent. Et ancorre que le roy ne montre poynt le trouver du tout si manvays, si esse que par se que y'ai seu, den [dans] son ceur il le trouve si manvays qui n'est possible de plus.

François de Lorraine, in einem parallelen Schreiben an den Schwager, konnte nur noch zustimmen, et confirmer mon advis et oppinion semblables ${ }^{108}$. Die letzte Möglichkeit, die der Herzogin von Guise zur Verhinderung dieser Heirat nun noch blieb, bestand darin, dem Bruder eine Alternative vorzuschlagen. Tatsăchlich arbeitete Anna mindestens seit Anfang 1557 am Zustandekommen der Verbindung Alfonsos mit einer Tochter oder mit der Schwester des Königs, furr die sich die Prinzessin schon sechs Jahre zuvor gemeinsam mit ihren Schwägern und der Herzogin von Valentinois eingesetzt hatte ${ }^{109}$. In ihrem Brief fordert sie den Bruder auf, sich noch vor seiner Hochzeit nach Frankreich $\mathrm{zu}$ begeben, pour des causes qui sont si grandes que ye ne les vous puis escripre, doch am Ende blieben alle Anstrengungen vergeblich. Gemäß dem Wunsche der Văter wurden Alfonso und die vierzehnjahrige Lucrezia de' Medici im Juni 1558 miteinander vermählt. Bis zum Tode Heinrichs im Juli und Ercoles im Oktober 1559 sollten die Beziehungen zwischen den beiden Fürsten gestört bleiben. Auch das immer schon schwierige Verhältnis Annas zu ihrem Bruder verbesserte sich nicht, im Gegenteil: In den folgenden vier Jahrzehnten sollten die Geschwister fast ständig im Streit liegen, sich um das Erbe des Vaters, der Mutter, der Schwester und des Bruders zanken.

${ }^{108}$ François de Lorraine an Alfonso d'Este, 22. Apr. 1558, Paris, in: ASM, Canc. duc., Cart. prin. est. 1568/13.

${ }^{109}$ Ercole d'Este an Cornelio Bentivoglio, 3. Mai 1551, in: ASM, Canc. duc., Amb. Francia 52; Giulio Alvarotti an Ercole d'Este, 14. Mărz 1551, in: FoNTANA, Renata di Francia, Bd. 2, S. 294; Charles de Lorraine an François de Lorraine, 2. Febr. 1557, in: LoRRAINE, Lettres, S. 254f.; ferner ROMIER, Origines, Bd. 1, S. 73-75. 
